\title{
HABILIDADES NO COGNITIVAS Y BRECHA DE GÉNERO SALARIAL EN EL PERÚ*
}

\author{
Gustavo Yamada \\ Centro de Investigación de la Universidad del Pacífico (CIUP) \\ Pablo Lavado \\ Centro de Investigación de la Universidad del Pacífico (CIUP) \\ Luciana Velarde \\ Universidad del Pacífico (UP)
}

\begin{abstract}
Resumen: En los últimos años, la literatura ha analizado la relación entre las habilidades cognitivas y no cognitivas y los resultados del mercado laboral. Más aún, se ha estudiado la correlación entre diferencias en estas habilidades y diferencias en salarios entre hombres y mujeres. No obstante, la mayoría de los estudios en torno al tema se ha enfocado en países desarrollados. El principal objetivo de este estudio es analizar el rol de las habilidades cognitivas y no cognitivas en la brecha salarial de género en el Perú. Para ello, proponemos uti-
\end{abstract}

Gustavo Yamada Fukusaki. Ph.D. en Economía, Universidad de Columbia, EE.UU. Profesor investigador del CIUP. Email: yamada_ga@up.edu.pe.

Pablo Lavado Padilla. Doctor en Economía, CEMFI, España. Profesor investigador del CIUP. Email: p.lavadopadilla@up.edu.pe.

Luciana Velarde Arrisueño. Magíster en Economía, Universidad del Pacífico, Perú. Profesional junior en educación del Banco Mundial. Email: lvelardearrisuen@, worldbank.org.

* Los autores agradecen los comentarios de Sergio Urzúa y de los participantes de "Conference on Skills, Education and Labor Market Outcomes", llevada a cabo en la Universidad de Maryland; de la conferencia "Inequalities in Children's Outcomes in Developing Countries", que tuvo lugar en la Universidad de Oxford, y de los seminarios de investigación del Banco Central de Reserva del Perú. 
lizar información longitudinal de medidas de habilidad para estimar componentes inobservables (o latentes) de ambas habilidades y analizar el rol de dichos componentes en el salario. Los resultados indican que existe una brecha salarial de género significativa en el Perú, que puede ser explicada por diferencias en el retorno de habilidades cognitivas latentes y por diferencias en la dotación de habilidades no cognitivas latentes. Luego de estimar un modelo conjunto de educación, empleo, ocupación y salarios, se observa que aun cuando las habilidades latentes explican la brecha de salarios, las mismas son más importantes para explicar la diferencia en la decisión de ocupación tomada entre hombres y mujeres.

Clasificación JEL: C31, C33, C35, J16, I21.

PALABRAs Clave: habilidades no cognitivas, habilidades latentes, brecha salarial de género.

RECIBIDO: julio 2013; ACEPTADO: enero 2014.

\section{NON-COGNITIVE SKILLS AND THE GENDER PAY GAP IN PERU}

ABSTRACT: Recently there has been growing interest in the relationship between cognitive and non cognitive abilities and labor market outcomes. A large literature provides evidence on the positive connection between gaps in abilities and gaps in wages between men and women. However, attention is focused on developed countries. The main objective of this paper is to identify latent abilities and explore their role in the gender wage gap in Peru. The main identification strategy relies on exploiting panel data information on test scores and arguing that time dependence across measures is due to latent abilities. Results show a significant gender wage gap in Peru and that even though when accounting for measured abilities differences in non cognitive abilities seem irrelevant, when accounting for differences in actual latent ability non cognitive abilities account for important inter-gender differences in the endowment and returns of abilities. Moreover, inter-gender differences in latent abilities play an important role not only in wage profiles, but in schooling, employment and occupation decisions.

JEL Classification: C31, C33, C35, J16, I21.

KEY WORDS: Non-cognitive skills, underlying ability, gender pay gap. RECEIVED: July 2013; ACCEPTED: January 2014. 


\section{INTRODUCCIÓN}

L a existencia de brechas salariales no es un tema nuevo, particularmente entre hombres y mujeres. A lo largo de los años, expertos en el tema han analizado los factores que podrían estar explicando ésta y otras brechas. Un tema que ha llamado la atención de estudiosos en este campo es el rol que juegan las diferencias en habilidades cognitivas y no cognitivas respecto de las brechas salariales. En particular, se ha documentado que diferencias en habilidades cognitivas entre hombres y mujeres se encuentran fuertemente relacionadas con diferencias en los salarios de los mismos (Neal y Johnson 1996; Ritter y Taylor 2011). En el caso de los hombres, el mayor nivel de este tipo de habilidades parece contribuir a sus mayores salarios. Más aún, las brechas en las habilidades cognitivas podrían contribuir a brechas salariales, no sólo por las diferencias en el nivel de estas habilidades, sino que también por diferencias en el retorno de las mismas ceteris paribus. Así, un punto adicional en una prueba cognitiva podría significar una mayor ganancia en términos de salario para un hombre que para una mujer.

Recientemente la literatura se ha enfocado en la relación entre las habilidades no cognitivas (ámbitos de la personalidad) y la productividad de los individuos (Heckman, Stixrud y Urzúa 2006). En cuanto a la relación entre las pruebas que miden habilidades no cognitivas e indicadores de mercado laboral, se ha encontrado que existe una relación positiva entre salarios y algunas medidas de habilidad no cognitiva (Fortin 2008; Grove et al. 2011; Cobb-Clark y Tan 2009). En cuanto a cómo se forma este tipo de habilidades, se ha cuestionado la idoneidad de las medidas comúnmente utilizadas, ya que parecen ser malas aproximaciones a las habilidades latentes o no observables (expresiones que usaremos como sinónimos en este artículo), debido a los posibles problemas de error en su medición y de causalidad reversa a las que son susceptibles (Heckman, Stixrud y Urzúa 2006). De hecho, uno de los principales factores que determinan los salarios son las habilidades latentes. Sin embargo, en una regresión de salarios en función de medidas de habilidad, parte de su efecto estaría capturando también el efecto de los años de educación, a través de su incidencia en las medidas de habilidad. No obstante, muy pocos estudios han analizado el rol de las habilidades no cognitivas latentes en la brecha salarial de género. Más 
aún, este tema en particular no ha sido abordado para países en vías de desarrollo ni, en particular, para la región de América Latina.

El principal objetivo de este documento es analizar el rol de las habilidades cognitivas y no cognitivas, latentes, en la brecha salarial de género, en un país en vías de desarrollo y por medio de aproximaciones de las habilidades no observables.

A fin de obtener una aproximación de las habilidades cognitivas y no cognitivas latentes, proponemos un procedimiento econométrico que permite estimar dichas variables, haciendo uso de un modelo similar al propuesto por Heckman, Stixrud y Urzúa (2006). Ellos plantean que de hallarse persistencia entre el puntaje obtenido por un mismo individuo en dos pruebas que midieran la misma habilidad, ésta podría estar capturando la habilidad latente correspondiente. A diferencia de dichos autores, nosotros explotaremos la disponibilidad de información de datos de panel para utilizar la persistencia a lo largo del tiempo en las pruebas de habilidades como fuente de identificación. Luego, complementaremos los estimados obtenidos con información de salarios, a fin de estimar el rol de las habilidades latentes en la brecha salarial de género. Partimos de un modelo simple al cual aplicamos el método de descomposición de Blinder-Oaxaca, para luego estimar un modelo conjunto de educación, empleo, ocupación y salarios. Asimismo, compararemos los resultados obtenidos haciendo uso de medidas de habilidad con aquellos obtenidos haciendo uso de las habilidades no observables estimadas como resultado del procedimiento econométrico anteriormente propuesto.

\section{REVISIÓN DE LA LITERATURA}

Durante décadas, los investigadores han centrado su atención en la relación entre el puntaje de pruebas de habilidad e indicadores del mercado laboral. Dichos estudios se concentran en el puntaje de evaluaciones de habilidades cognitivas, como tests de razonamiento matemático y verbal, entre otros. Murnane, Willett y Levy (1995) midieron el rol de las habilidades matemáticas de jóvenes próximos a graduarse de la secundaria en sus salarios a los 24 años y encontraron una relación positiva y creciente de dichas habilidades en los salarios (especialmente cuando las habilidades fueron medidas próximas a la graduación). En 
un estudio más reciente, Cunha et al. (2006) propusieron que la habilidad cognitiva afecta la probabilidad de alcanzar un mayor nivel educativo y el retorno económico del mismo.

Recientemente se ha incrementado el interés por analizar el rol de las habilidades no cognitivas y su impacto en variables asociadas al mercado laboral. Trabajos precursores como los de Bowles y Gintis (1976), Edwards (1976) y Klein, Spady y Weiss (1991) mostraron que habilidades no cognitivas como la dependencia y persistencia son altamente valoradas por los empleadores. Estudios más recientes como el de Heckman, Stixrud y Urzúa (2006) apoyan la existencia de dicha relación, al encontrar una relación positiva entre el puntaje de pruebas que miden habilidades no cognitivas e indicadores asociados al mercado laboral.

En cuanto a la contribución de las diferencias en habilidades a las brechas en el mercado laboral, gran parte de los estudios abordan el rol de las habilidades cognitivas en brechas salariales raciales o de género (Neal y Johnson 1996; Ritter y Taylor 2011). Pocos estudios, sin embargo, abordan la contribución de habilidades no cognitivas a las brechas salariales de género.

En este sentido, Fortin (2008) investigó el impacto de aspectos de la personalidad como autoestima, locus de control, la importancia del dinero/trabajo y la importancia de las personas/familia en la brecha salarial de género. Haciendo uso de dos encuestas longitudinales, la NLS72 y la NELS88, encontró que factores no cognitivos explican una parte pequeña pero importante - alrededor de 2 puntos en el logaritmo del salario - de la brecha salarial de género entre trabajadores de treinta años. En particular, en cuanto a la importancia otorgada al "dinero/ trabajo" y a las "personas/familia" respecto de la autoestima y confianza, se observó que mientras los hombres suelen ser más ambiciosos y valorar más aquellos empleos que ofrecen salarios altos, las mujeres suelen optar por trabajos con un componente altruista.

Grove et al. (2011) exploraron el poder explicativo de variables no cognitivas y variables de capital humano en la brecha salarial de profesionales con MBA. Haciendo uso de una encuesta longitudinal de individuos que se registraron para la prueba GMAT entre 1990 y 1998 , encontraron que el 82 por ciento de la brecha salarial de género es explicada por habilidades no cognitivas y preferencias relacionadas con la 
familia, carrera y empleo. Asimismo, al parecer las mujeres en la muestra experimentaban una penalidad en el salario por revelar preferencias hacia trabajos altruistas, los cuales en promedio otorgan menores salarios. Los hombres parecían atribuir una mayor importancia a la riqueza, pero ello no estuvo asociado con la brecha salarial entre ambos. La brecha estuvo explicada principalmente por la experiencia educativa y la tenencia de empleo por parte de los hombres.

Cobb-Clark y Tan (2009) evaluaron si las habilidades no cognitivas de hombres y mujeres ejercen influencia sobre su ocupación y si, por tanto, contribuyen a la disparidad en salarios relativos. Utilizando información de empleados (no autoempleados) de 25 a 65 años, contenida en la encuesta de Hogares, Ingreso y Dinámica Laboral en Australia (HILDA) de 2001 y 2006, encontraron una relación entre el nivel de habilidades no cognitivas y la ocupación en la que dichos empleados laboraban. No obstante, si bien la brecha salarial de género fue de 0,143 (en términos del logaritmo del salario), el 96,6 por ciento se atribuyó a diferencias en el salario de hombres y mujeres, considerando que eran empleados en la misma ocupación. Por tanto, el componente más importante de la brecha salarial de género ocurre al interior de cada ocupación y permanece aún inexplicado.

Gran parte de los estudios en torno al rol de las pruebas que evalúan habilidades cognitivas y no cognitivas en indicadores de mercado laboral se han realizado para países desarrollados. A nuestro entender, muy pocos han abordado el tema para países en desarrollo y, en particular, para países de la región latinoamericana.

Bassi y Galiani (2009) utilizaron una encuesta nacional con información sobre adultos de 25 a 30 años, a fin de explorar el rol que ejercen las pruebas que miden habilidades cognitivas y no cognitivas sobre el logaritmo de los salarios. Los autores encontraron coeficientes significativos para ambos tipos de habilidad, pero también que los mismos se reducen luego de controlar por el nivel educativo de los individuos. Este resultado probablemente esté asociado al hecho de que al utilizar medidas de habilidad explícitas (a diferencia de habilidades latentes) el efecto de los años de educación genera sesgo en los coeficientes de dichas medidas, por ejemplo, en regresiones de salarios que no controlan por años de educación. 
Díaz et al. (2012) estimaron retornos a la educación, habilidades cognitivas y no cognitivas en el Perú utilizando la Encuesta Nacional de Habilidades y Mercado Laboral (Enhab) — que comprende una muestra de personas en edad de trabajar - y mediante una aproximación de variables instrumentales para abordar los problemas asociados a la endogeneidad de los años de educación presentes en la ecuación de salarios. Los autores hallaron que el mercado laboral peruano valora la educación y las habilidades cognitivas y no cognitivas. En particular, concluyeron que el incremento de una desviación estándar en los años de educación se encuentra asociado con un incremento de 15 por ciento en los salarios, mientras que cambios similares en el nivel de habilidades cognitivas y no cognitivas se encuentran asociados con incrementos de 9 por ciento y 5 a 8 por ciento en los salarios, respectivamente.

Urzúa et al. (2009) profundizaron en el análisis de discriminación de género en el mercado laboral para el caso de Chile, utilizando información sobre indicadores de mercado laboral, logro y desempeño educativo y otras variables relacionadas con el entorno familiar de cada individuo. Los autores trabajaron sobre la base de estudios previos que abordan la estimación de modelos de mercado laboral con múltiples fuentes de heterogeneidad no observada, heterogeneidad generada por habilidades cognitivas y no cognitivas. Sin embargo, por limitaciones en la disponibilidad de la información sólo consideraron una fuente de heterogeneidad no observada; es decir, capturaron el efecto de las habilidades cognitivas y no cognitivas en un solo componente, como una sola variable. Los resultados obtenidos sugieren la existencia de brechas de género en variables tales como experiencia, empleo, horas trabajadas y salarios por hora que no pueden ser explicadas por características observables o mecanismos de selección que generan endogeneidad. No obstante, hallaron evidencia de que las mujeres que tienen bajos niveles educativos suelen ser víctimas de discriminación salarial. Hasta donde tenemos conocimiento, este estudio es el único que aborda, en la brecha salarial de género, el rol de habilidades cognitivas y no cognitivas, latentes, en un país en vías de desarrollo.

De acuerdo con muchos de los estudios presentados líneas arriba, un segundo tema de interés es el proceso de formación de las habilidades cognitivas y no cognitivas. Heckman, Stixrud y Urzúa (2006) argumentan que las pruebas que miden este tipo de habilidades no reflejan 
el "verdadero" nivel de habilidad (lo denominado "habilidad latente"), ya que, al ser función del nivel educacional, se encuentran medidas con error. Por tanto, utilizar el puntaje de dichas pruebas en regresiones de salarios y años de educación es problemático. Al controlar por el nivel educacional, tanto las habilidades cognitivas como las no cognitivas predicen los salarios. Sin embargo, el nivel educacional es una variable de elección y, por tanto, implica problemas de endogeneidad que deben ser considerados. Omitir el nivel educacional de una ecuación de salarios incrementa la correlación entre ambos tipos de habilidades y el salario percibido. Los efectos estimados comprenden tanto el efecto directo de las habilidades en los salarios (sobre la productividad), como el indirecto (a través del nivel educacional). No obstante, existe una importante diferencia entre pruebas que miden las habilidades no cognitivas y cognitivas, como las de Coeficiente Intelectual (CI) y pruebas que miden logros de aprendizaje. Si bien el CI tiende a establecerse alrededor de los 8 años de edad, los resultados de pruebas de logros de aprendizaje han evidenciado ser cambiantes y crecientes según el nivel educacional adquirido.

Hansen et al. (2004) desarrollaron dos métodos para estimar el efecto del nivel educacional en las pruebas de logros de aprendizaje que controlan por la endogeneidad asociada. Plantean que tanto el nivel educacional adquirido como los puntajes de pruebas de habilidades son generados por un componente común no observado: las habilidades latentes. De esta manera, encontraron que el efecto del nivel educacional en el puntaje de pruebas de habilidad es lineal o proporcional a través de niveles educativos y mayor para bajos niveles de habilidad. Alcanzar un mayor nivel educativo incrementa entre 2 y 4 puntos porcentuales el puntaje obtenido en la prueba Armed Forces Qualification Test (AFQT), considerada por el estudio en cuestión. Los autores contribuyeron a estimar el impacto del nivel educacional sobre las habilidades medidas en varios quintiles de la distribución de habilidades latentes. Asimismo, presentaron evidencia según la cual la medida de CI utilizada por Herrnstein y Murray (1994) se encuentra fuertemente afectada por el nivel educativo de los individuos. Modelaron los puntajes de pruebas de habilidades como función de las habilidades latentes (y de otros factores) y modelaron también el nivel educacional como función de las habilidades latentes (y de otros determinantes). Asimismo, consideraron efectos de truncamiento (en pruebas sencillas, el puntaje máximo puede ser alcanza- 
do por niños con distintos niveles de habilidad) y endogeneidad del nivel educativo (elecciones de fecha de entrada y salida del sistema educativo).

Helmers y Patnam (2011) investigaron los factores que determinan los puntajes en pruebas de habilidades cognitivas y no cognitivas para niños en Andhra Pradesh, India, utilizando una base de datos que contiene información sobre dos cohortes de niños de hasta 12 años de edad. Aprovechando la disponibilidad de datos de panel estimaron un modelo de Relaciones Estructurales Lineales (Lisrel), el que permite estimar habilidades latentes (cognitivas y no cognitivas) e inversión familiar, y además relacionar estas variables a otras características observables asociadas al niño, sus padres y el hogar al que pertenece. Trabajaron sobre la base de lo desarrollado por Cunha y Heckman (2007), con el fin de examinar la dinámica que gobierna la formación de habilidades cognitivas y no cognitivas, y la relación entre ambas a lo largo del tiempo. En particular, centraron su atención en explorar los determinantes en el proceso de formación de ambos tipos de habilidades. Los autores encontraron evidencia a favor de la importancia de la inversión familiar (cuidado por parte de los padres durante el embarazo y los primeros meses del niño) y la salud de los niños durante el primer año de edad.

De esta manera, la principal contribución del presente estudio es analizar el rol de las habilidades latentes cognitivas y no cognitivas, como también del retorno de las mismas - en términos de salarios-, en la brecha salarial de género, todo esto en un país en vías de desarrollo. Para ello, uno de nuestros principales objetivos es estimar las habilidades latentes, que son no observables para el econometrista pero persistentes en el tiempo.

\section{MODELO}

El modelo planteado está construido sobre la base de lo propuesto por Heckman, Stixrud y Urzúa (2006), Cunha y Heckman (2007) y Cunha, Heckman y Schennach (2010). El modelo plantea que existen dos factores subyacentes: la habilidad latente cognitiva y la habilidad latente no cognitiva. Así, controlando por factores observables, estos factores son capaces de explicar la dependencia entre decisiones y resultados. Ambos factores son conocidos por cada individuo, pero no para el econometrista. Asimismo, el nivel de habilidades latentes se encuentra fijo para el momento en que el individuo toma decisiones en el mercado laboral. 
La estrategia de identificación es similar a la planteada en Heckman, Stixrud y Urzúa (2006). La habilidad cognitiva latente $\left(f^{\mathrm{C}}\right)$ afecta sólo a la medida de habilidad cognitiva $(C)$, y la habilidad no cognitiva latente $\left(f^{\mathrm{N}}\right)$ afecta sólo a la medida de habilidad no cognitiva $(N)$.

$$
\begin{gathered}
C=\beta_{C} X_{C}+\alpha_{C} f^{C}+e_{C} \\
N=\beta_{N} X_{N}+\alpha_{N} f^{N}+e_{N}
\end{gathered}
$$

El modelo se sustenta en el hecho de que, condicional a la información contenida en el vector de variables $\mathrm{X}$, la dependencia temporal entre las medidas de habilidad es atribuible a las habilidades latentes. Controlar por esta dependencia implica controlar la endogeneidad del modelo. Heckman, Stixrud y Urzúa (2006) estiman la distribución de habilidades latentes aprovechando la disponibilidad de dos o más medidas de habilidad. En contraste, la estrategia de especificación que utilizaremos aprovecha la disponibilidad de datos de panel para las medidas de habilidad; en concreto, tener información sobre la misma medida de habilidad en dos momentos del tiempo. Asimismo, asumimos un modelo lineal en los parámetros. No obstante, ello puede ser interpretado como una aproximación de un modelo de comportamiento más flexible, tal como en Heckman, Stixrud y Urzúa (2006).

$\mathrm{Al}$ analizar el rol de las habilidades en la brecha salarial de género, se debe tomar en cuenta que parte de lo que podría interpretarse como discriminación podría en realidad deberse a la dinámica comprendida en la elección de ocupaciones; hombres y mujeres podrían autoseleccionarse hacia ocupaciones que premian distintos tipos de habilidad. Asimismo, si bien controlar por los años de educación de un individuo en una ecuación de salarios genera endogeneidad, se debe considerar el rol de las habilidades en la elección de un nivel educacional. Por tanto, de manera similar a lo aplicado en Heckman, Stixrud y Urzúa (2006), trabajaremos sobre la base de un modelo que considere las decisiones de educación, empleo y ocupación.

El modelo tiene cuatro etapas, a fin de controlar por la correlación de los errores de cada decisión. El individuo elige un nivel educativo considerando el acervo de habilidades latentes; más tarde, dado el nivel educativo y sus habilidades latentes, él mismo elige participar o no del mercado laboral. Así, luego de tomar la decisión de participar, el indivi- 
duo debe elegir el tipo de ocupación en el que desea trabajar, considerando su nivel de habilidades, y finalmente, dadas todas las elecciones tomadas hasta el momento y el acervo de habilidades, al individuo le es asignado un determinado salario. A continuación se presenta el modelo de manera formal.

\subsection{Modelo de educación}

Cada individuo elige el nivel de educación que maximiza su beneficio tomando en cuenta el nivel de habilidades cognitivas y no cognitivas latentes que tiene. Así, considerando una especificación lineal en los parámetros, es el beneficio neto asociado con haber logrado un determinado nivel educativo:

$$
I_{s}=\beta_{S} X_{S}+\alpha_{s}^{C} f^{C}+\alpha_{s}^{N} f^{N}+e_{s}
$$

donde $s$ es el nivel educativo elegido por el individuo entre dos opciones: secundaria completa como máximo nivel educativo o, al menos, un año de educación superior. $X_{s}$ es un vector de variables observables que afectan la decisión de educación, $\beta_{s}$ es el vector de parámetros asociados, $\alpha_{S}^{C}$ y $\alpha_{S}^{N}$ son las ponderaciones de las habilidades cognitivas $\mathrm{y}$ no cognitivas latentes, respectivamente, $\mathrm{y} e_{S}$ es representa un error idiosincrásico que se asume como independiente de $f^{N}, f^{C}$ y $X_{S}$. Los términos de error correspondientes a ambos niveles educativos son independientes.

\subsection{Modelo de empleo}

Luego de elegir el nivel educativo y considerando el resultado de dicha elección, el individuo debe tomar la decisión acerca de participar o no en el mercado laboral. Así, el individuo decidirá participar si el beneficio neto asociado es mayor a que no participara, considerando también su nivel de habilidades latentes. Así, es el beneficio asociado a trabajar en el que se asume una especificación lineal en los parámetros:

$$
I_{E}=\beta_{E} X_{E}+\alpha_{E}^{C} f^{C}+\alpha_{E}^{N} f^{N}+e_{E}
$$


donde $E$ es la decisión tomada por el individuo: trabajar o no trabajar. $X_{E}$ es un vector de variables observables que afectan la decisión de empleo, $\beta_{E}$ es el vector de parámetros asociados, $\alpha_{E}^{N}$ y $\alpha_{E}^{C}$ son las ponderaciones de las habilidades cognitivas y no cognitivas latentes, respectivamente, y $e_{E}$ representa un error idiosincrásico que se asume como independiente de $f^{N}, f^{C}$ y $X_{E}$. Los términos de error correspondientes a ambas opciones laborales (empleo o desempleo) son independientes.

\subsection{Modelo de ocupación}

Una vez tomada la decisión de participar del mercado laboral, el individuo debe elegir en qué ocupación desempeñarse. Así, considerando las decisiones tomadas hasta el momento y su nivel de habilidades latentes, decidirá una de dos opciones de ocupación: la primera, una ocupación que requiere de capacitación especializada (denominada "white collar"); la segunda, una que no la requiera y esté más orientada al trabajo manual ("blue collar"). Así, $I_{0}$ es la utilidad asociada a trabajar en una ocupación determinada:

$$
I_{0}=\beta_{0} X_{0}+\alpha_{0}^{C} f^{C}+\alpha_{0}^{N} f^{N}+e_{0}
$$

donde 0 representa la ocupación elegida por el individuo. $X_{0}$ es un vector de variables observables que afectan la decisión de ocupación, $\beta_{o}$ es el vector de parámetros asociados, $\alpha_{0}^{C}$ y $\alpha_{0}^{N}$ son las ponderaciones de las habilidades cognitivas y no cognitivas latentes, respectivamente, y $e_{0}$ representa un error idiosincrásico que se asume como independiente de $f^{N}, f^{C}$ y $X_{0}$. Los términos de error correspondientes a ambas opciones ocupacionales son independientes.

\subsection{Modelo de salarios}

Finalmente, el salario que recibe el individuo es producto de las decisiones previas de educación, empleo y ocupación, así como el nivel de habilidades cognitivas y no cognitivas latentes del individuo hasta ese momento. Por tanto, la ecuación de salarios sería:

$$
Y=\beta_{Y} X_{Y}+\alpha_{Y}^{C} f^{C}+\alpha_{Y}^{N} f^{N}+e_{Y}
$$


donde $X_{Y}$ es un vector de características observables, $\beta_{Y}$ es el vector de retornos asociados, $\alpha_{Y}^{C}$ y $\alpha_{Y}^{N}$ son las ponderaciones de las habilidades cognitivas y no cognitivas latentes, respectivamente, y $e_{Y}$ representa un error idiosincrásico que se asume como independiente de $f^{N}, f^{C}$ y $X_{Y}$.

De esta manera, se hace posible desagregar el efecto de las habilidades latentes en los salarios, tomando en cuenta el efecto que las mismas podrían tener en las decisiones previas a la asignación del salario en sí mismo, ello de manera diferenciada entre hombres y mujeres.

\section{Fuentes de la muestra}

Con el objetivo de estimar el efecto de las habilidades en la brecha salarial de género, es necesario contar con una base de datos que contenga información sobre ambas variables para un mismo individuo. Así también, no sólo es necesario contar con información sobre medidas de habilidad; a fin de aislar la denominada habilidad latente, la estrategia de identificación propuesta requiere información sobre las medidas de habilidad de un individuo en distintos periodos. Debido a la ausencia de una única fuente de información que cumpla con dichas características -información acerca de salarios y habilidades en distintos periodos para el mismo conjunto de individuos-, aplicaremos un procedimiento econométrico que permitirá combinar la información contenida en dos bases de datos.

La primera base de datos corresponde a la recogida en el Estudio de Niños del Milenio (ENM). Ésta comprende información longitudinal para dos cohortes de niños ("cohorte joven" y "cohorte adulta") y para cada uno de cuatro países: Etiopía, India (Andhra Pradesh), Perú y Vietnam. En Perú la muestra representa al 95 por ciento de los niños (excluye al 5 por ciento que pertenece a las familias con mayores ingresos). Los niños y sus cuidadores principales fueron encuestados tres veces: años 2002, 2006 y 2009. La encuesta recoge información sobre aspectos relacionados con el desarrollo de los niños, medidas de habilidad cognitiva y aspectos de su personalidad (actitudes y aspiraciones), medidas antropométricas y un conjunto de otras características a nivel individual y de hogares. En particular, incluye características tales como el nivel socioeconómico de las familias (percibido por el niño), 
índices de riqueza, gasto en consumo y medidas de las habilidades de los cuidadores principales, entre otros.

Con el fin de analizar la distribución de habilidades entre niños peruanos, centramos nuestra atención en los 700 niños de la "cohorte adulta", los mismos que tenían 8 años en la primera ronda del estudio (2002). Asimismo, mantenemos la submuestra de niños con información disponible para los ítemes relacionados con medidas de habilidad (cognitiva y no cognitiva) e información individual relevante para las rondas 2 y 3 , cuando los niños de la "cohorte adulta" tenían 12 y 15 años de edad, respectivamente" ${ }^{1}$. Finalmente, dado que se buscará hacer un paralelo entre la información contenida en la ENM y la Enhab (la cual contiene información de salarios para personas en edad de trabajar que viven en zonas urbanas), trabajamos solamente con los niños de la "cohorte adulta" que viven en zonas urbanas. La muestra final consta de 349 niños.

La muestra con la que trabajaremos en adelante se encuentra distribuida entre niños y niñas (165 y 184 , respectivamente), que tienen una edad promedio de 149 meses y un promedio de seis años de educación durante la ronda 2. La tabla 1 presenta estadísticos descriptivos de las principales variables de interés para ambas rondas, así como información sobre la lengua materna y el nivel educacional de los padres en la ronda 1 (lo que denominaremos "características permanentes"). Entre estas variables se encuentran: la puntuación directa ${ }^{2}$ ("raw score") del Test de Vocabulario en Imágenes de Peabody (TVIP) como medida de habilidad cognitiva; un índice de autoeficacia y un índice de autoestima, estas últimas a modo de medida de habilidad no cognitiva ${ }^{3}$. Dicho

\footnotetext{
${ }^{1}$ En la ronda 1 no se recogió información sobre habilidades cognitivas para la cohorte adulta ni en las rondas 2 o 3 para la cohorte joven.

${ }^{2}$ La puntuación directa es el resultado de la diferencia entre el "ítem tope" - la última pregunta a partir de la cual el niño falla de manera sistemática- y el número de errores cometidos por el niño antes del "ítem tope".

${ }^{3}$ Ambos índices — uno para cada aspecto de la personalidad - consisten en el promedio del puntaje asignado según el grado de acuerdo o desacuerdo de los niños respecto de los enunciados correspondientes a cada habilidad, que luego estandarizamos para facilitar la interpretación al realizar las estimaciones. Para el caso de autoestima, los enunciados incluidos en el ENM se centraron en dimensiones positivas y negativas del orgullo y la vergüenza sobre la base de la Escala de Autoestima de Rosenberg, la cual se centra en dimensiones de las circunstancias diarias de la vida del niño. Para el caso de autoeficacia, centramos la atención en
} 


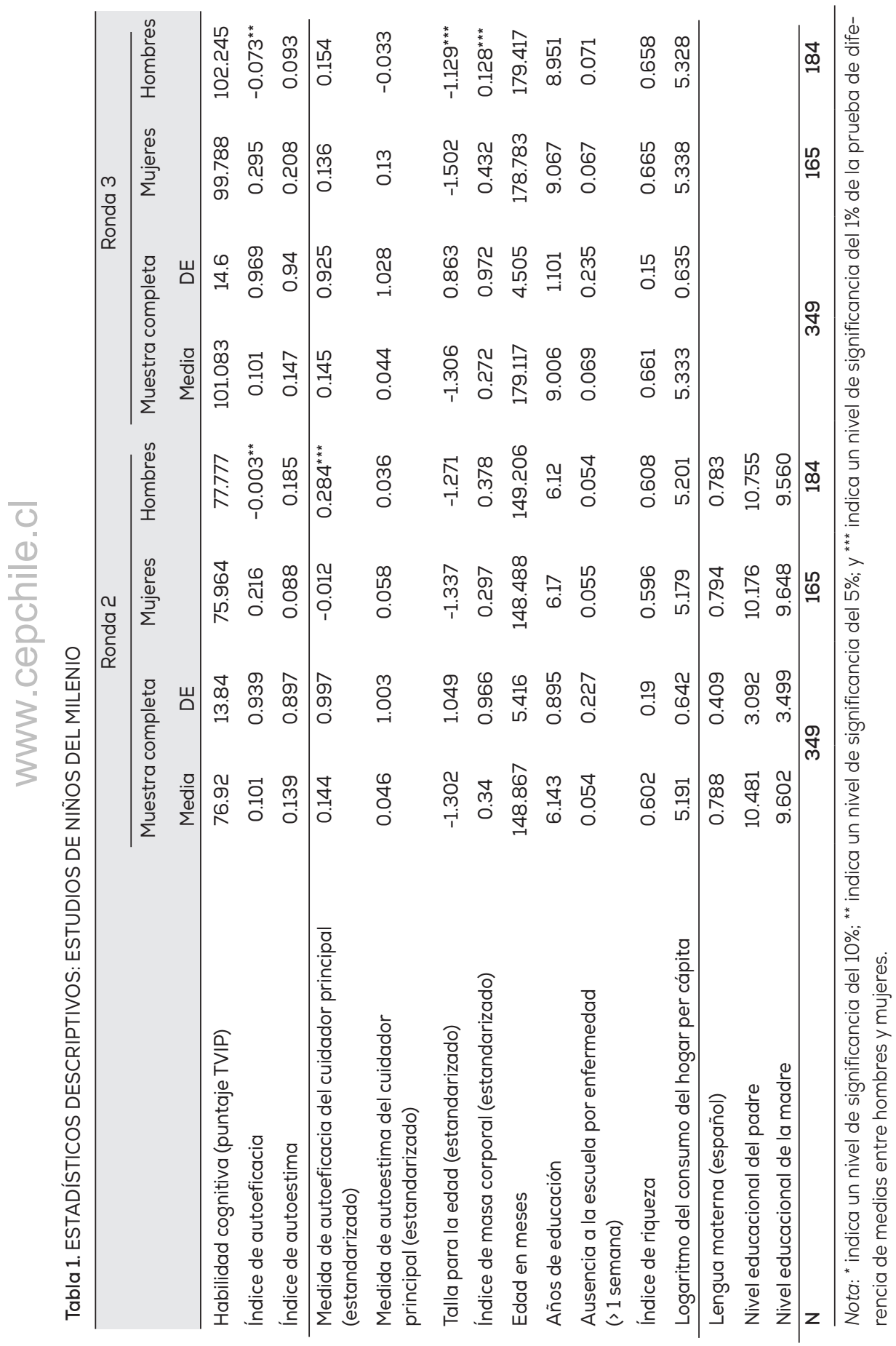


estudio cuenta con información de medidas de habilidad no cognitiva no sólo para los niños, sino que también la correspondiente a los cuidadores principales de dichos niños (ambos índices se construyeron de la misma manera en ambos casos). En las dos rondas, si bien las mujeres alcanzan un puntaje menor al promedio en medidas de habilidad, lo mismo sucede para los hombres en el índice de autoeficacia, mientras que los resultados son mixtos para el caso de autoestima. No parece haber diferencias importantes entre géneros en cuanto a características del hogar y la familia. Las medidas de habilidad no cognitivas de los cuidadores principales difieren entre niños y niñas; los primeros evidencian altos niveles de autoeficacia durante la ronda 2 y bajos niveles de autoestima durante la ronda 3. Asimismo, diferencias importantes surgen entre rondas.

La segunda base de datos corresponde a una novedosa encuesta de hogares recogida por el Banco Mundial en el año 2010, que contiene información de salarios y características individuales, así como medidas de habilidad cognitiva y no cognitiva para una muestra de individuos en edad de trabajar (14-50 años). Se trata de la Encuesta Nacional de Habilidades y Mercado Laboral (Enhab), representativa a nivel del Perú urbano. Dicha base de datos contiene información acerca de las condiciones de vida, información demográfica, logro académico, empleo/ salarios e información nueva sobre medidas de habilidad cognitiva y no cognitiva, trayectoria educativa, participación temprana en el mercado laboral y características familiares. Las habilidades cognitivas fueron medidas por medio de pruebas que evalúan capacidades numéricas y de resolución de problemas, memoria de trabajo, fluidez verbal y de lenguaje receptivo. Las habilidades no cognitivas fueron medidas de acuerdo con las escalas GRIT (Duckworth et al. 2007) y con los Cinco

cinco enunciados: "Si me esfuerzo, puedo mejorar mi situación de vida"; "Otras personas en mi familia toman las decisiones acerca de cómo invierto mi tiempo"; "Me gusta formular planes acerca de mis estudios y trabajo futuros"; "(No) puedo elegir en qué empleo trabajar" y "Si estudio con mucho empeño, seré recompensado con un mejor empleo en el futuro". El grado de acuerdo con dichos enunciados se mide en una escala de cuatro puntos de Likert, que oscila desde el fuerte acuerdo hasta el fuerte desacuerdo. A fin de promediar el puntaje asignado a cada uno de los enunciados, se mantuvieron los enunciados con un enfoque positivo (por ejemplo: "Me gusta formular planes acerca de mis estudios y trabajo futuros") y se invirtió la escala para aquellos con enfoque negativo (por ejemplo: “(No) puedo elegir en qué empleo trabajar"). 
Grandes Factores de la Personalidad (Goldberg 1990). Para el análisis que realizaremos, concentramos nuestra atención en siete medidas, los valores estandarizados de cada uno de los cinco factores de la personalidad (estabilidad emocional, extroversión, amabilidad, cooperación, actitud concienzuda (fuerte) y apertura), una medida compuesta de los elementos de GRIT, y una medida compuesta de las habilidades cognitivas. Algunas de las características individuales comprendidas son el pasado educativo del individuo, características familiares y estatus

Tabla 2. ESTADÍSTICOS DESCRIPTIVOS: ENCUESTA NACIONAL DE HABILIDADES Y MERCADO LABOR (ENHAB)

\begin{tabular}{|c|c|c|c|c|c|}
\hline & $\mathrm{N}$ & Media & DS & Mujeres & Hombres \\
\hline $\begin{array}{l}\text { Habilidad cognitiva } \\
\text { (Puntaje TVIP) }\end{array}$ & 2421 & 42.001 & 14.932 & 40.103 & $45.039^{* * *}$ \\
\hline Consistencia / Interest & 2421 & -0.004 & 0.998 & 0.036 & $-0.069^{\star \star *}$ \\
\hline Persistencia / Esfuerzo & 2421 & 0.001 & 1.001 & -0.046 & $0.076^{\star \star *}$ \\
\hline GRIT (estandarizado) & 2421 & -0.002 & 0.997 & -0.009 & 0.008 \\
\hline Extroversión & 2421 & 0.002 & 1 & -0.047 & $0.082^{* * *}$ \\
\hline Amabilidad & 2421 & 0.002 & 1.006 & 0.039 & $-0.058^{* *}$ \\
\hline Cooperación & 2421 & 0.006 & 0.992 & 0.051 & $-0.065^{* * *}$ \\
\hline Actitud concienzuda (fuerte) & 2420 & 0.000 & 1.001 & 0.056 & $-0.092^{\star \star \star}$ \\
\hline Estabilidad emocional & 2415 & 0.013 & 0.997 & -0.072 & $0.149^{* * *}$ \\
\hline Apertura & 2415 & -0.004 & 0.999 & -0.059 & $0.085^{\star \star \star}$ \\
\hline Logaritmo del salario por hora & 4063 & 1.316 & 0.815 & 1.166 & $1.408^{* * *}$ \\
\hline Salario mensual & 4063 & 972.155 & 1284.643 & 738.7 & $1116.299^{* * *}$ \\
\hline Salario por hora & 4063 & 5.264 & 6.55 & 4.638 & $5.651^{\star \star \star}$ \\
\hline Horas semanales de trabajo & 4063 & 51.127 & 18.482 & 46.339 & $54.084^{* * *}$ \\
\hline Experiencia & 4063 & 25.43 & 13.582 & 25.314 & 25.502 \\
\hline Edad & 7499 & 33.514 & 15.282 & 33.305 & 33.736 \\
\hline Años de educación & 7457 & 10.701 & 3.373 & 10.524 & $10.890^{* * *}$ \\
\hline Lengua materna (español) & 7499 & 1.008 & 0.142 & 1.007 & 1.009 \\
\hline Nivel educacional del padre & 7499 & 4.955 & 2.373 & 4.947 & 4.963 \\
\hline Nivel educacional de la madre & 7499 & 4.287 & 2.333 & 4.253 & 4.324 \\
\hline
\end{tabular}

Nota: ${ }^{*}$ indica un nivel de significancia del $10 \%$; ${ }^{* *}$ indica un nivel de significancia del $5 \%$; $\mathrm{y}^{* * *}$ indica un nivel de significancia del $1 \%$ de la prueba de diferencia de medias entre hombres y mujeres. Los estadisticos descriptivos fueron calculados considerando la totalidad de individuos que cuentan con información disponible para la variable correspondiente. 
socio-económico (educación y ocupación de los padres, tamaño de la familia, información acerca del acceso y particularidades de la escuela a la que asistieron los padres, entre otros).

En términos generales, los individuos en la base de datos se encuentran distribuidos de manera similar entre hombres y mujeres, tienen en promedio 33 años de edad, ganan un salario de 1.000 nuevos soles constantes del año 2010 (aproximadamente 350 USD), trabajan 51 horas a la semana y han concluido estudios de secundaria. La tabla 2 ilustra estadísticos descriptivos de las principales variables a considerar, así como la diferencia entre hombres y mujeres en cada una. Entre ellos, se encuentra el hecho de que el hombre representativo de la muestra tiene salarios mayores (mensuales y por hora), trabaja más horas y tiene niveles más altos de habilidades cognitivas que la mujer representativa de la muestra. Sin embargo, los resultados son mixtos para el caso de las habilidades no cognitivas. Mientras las mujeres muestran actitudes más consistentes, amables, cooperativas y concienzudas, los hombres presentan actitudes más persistentes, extrovertidas, emocionalmente estables y abiertas. La muestra con la que trabajaremos en adelante es la conformada por aquellos individuos para los cuales se cuenta con información sobre medidas de habilidad (puntaje de pruebas), salarios y las principales características individuales $(\mathrm{N}=2421)^{4}$.

\section{Implementación econométrica}

El primer objetivo de este documento es identificar la contribución de las habilidades a la brecha salarial de género. En el afán de identificar el rol de las habilidades en los salarios, la ecuación que se desearía estimar es:

$$
L n W_{i}=\alpha+\gamma S_{i}+\beta_{A} A_{i}+\mu_{i t}
$$

donde $\operatorname{Ln} W_{i}$ es el logaritmo de los salarios, $S_{i}$ representa los años de educación y $A_{i}$, la habilidad innata del individuo. Sin embargo, la poca disponibilidad de datos acerca de las habilidades innatas lleva a omitir

${ }^{4}$ No existen diferencias significativas en las principales variables de interés entre la población encuestada en la Enhab y la muestra con información disponible para las variables relevantes. 
$A_{i}$, generando endogeneidad en la ecuación de salarios. Si se asume que las habilidades generan incrementos en el salario y que las habilidades están positivamente relacionadas con los años de educación, $\gamma$ estaría sobreestimando el verdadero valor del parámetro. La literatura empírica ha abordado este tema mediante la inclusión de medidas de habilidad (puntaje de pruebas que miden habilidades) y, por tanto, estimando la siguiente ecuación:

$$
L n W_{i}=\alpha+\gamma S_{i}+\beta_{T} T_{i}+v_{i t}
$$

donde $T_{i}$ representa el puntaje de pruebas estandarizadas que miden habilidades cognitivas o no cognitivas. Esta especificación es problemática, ya que es probable que los puntajes de pruebas de habilidades no sólo estén determinados por las habilidades innatas (cuyo efecto desearíamos aislar), sino que también por el nivel educacional del individuo, lo cual genera un problema, pues ambos factores estarían asociados con incrementos en los salarios. Por tanto, $\beta_{T}$ estaría capturando parcialmente el efecto indirecto de los años de educación en los salarios, a través de su efecto en las medidas de habilidad e impidiendo obtener el verdadero efecto de los años de educación en los salarios.

En este sentido, proponemos aplicar un procedimiento econométrico que permita estimar esta habilidad (latente) $-A_{i}-\mathrm{e}$ identificar cada uno de los efectos deseados en los salarios. Para ello, explotaremos la información longitudinal de medidas de habilidad contenida en el ENM para estimar proxies de las habilidades cognitivas y no cognitivas latentes. Luego, proponemos un método de imputación para predecir los efectos fijos estimados con la muestra del ENM en la muestra de la Enhab. Al complementar estas habilidades latentes predichas con la información de salarios recogida para la Enhab, será posible analizar el rol de las habilidades cognitivas y no cognitivas latentes en los salarios. Por tanto, la implementación econométrica que aplicaremos se divide básicamente en dos etapas: (i) la estimación de las habilidades latentes para la muestra del ENM, y (ii) la estimación del efecto de las habilidades latentes (predichas) en los salarios para la muestra Enhab.

En la primera etapa trabajaremos con la base de datos del ENM. En primer lugar, haremos uso de la variación (de la ronda 2 a la ronda 3) en las medidas de habilidad y los años de educación entre los niños 
en la muestra del ENM para recuperar los efectos fijos (no observables). En particular, intentaremos explicar la variación de las tres medidas de habilidad, dos habilidades no cognitivas (autoestima y autoeficacia) y una habilidad cognitiva (Test de Vocabulario e Imágenes Peabody, TVIP). El procedimiento de identificación requiere que se controle por cambios en las características que puedan haber variado entre los 12 y 15 años y puedan explicar gran parte de la variación en las medidas de habilidad de los niños durante ese mismo período. De esta manera, será posible explicar los cambios en las medidas de habilidad y aislar los efectos fijos no observables.

$$
\Delta M A_{i t}=\gamma_{X} \Delta X_{i t}+\Delta \mu_{i t}
$$

Luego, estimaremos la correlación entre las características que se mantienen constantes a lo largo de la vida del niño y los efectos fijos previamente hallados. Para ello, capturaremos el efecto fijo o componente no observable de cada tipo de habilidad haciendo uso de los estimados obtenidos en la primera etapa, para predecirlos a continuación haciendo uso del valor promedio de los regresores en las rondas 2 y 3 y, luego, desviando el valor predicho de la medida de habilidad respecto del valor observado correspondiente.

$$
\begin{aligned}
& \hat{M} A_{i t}=\hat{\gamma}_{0}+\hat{\gamma}_{X} X_{i t} \\
& \hat{L A_{i}}=\frac{1}{2}\left[\left(M A_{i 1}-\hat{M} A_{i 1}\right)+\left(M A_{i 2}-\hat{M} A_{i 2}\right)\right]
\end{aligned}
$$

Los valores hallados representarán proxies de las habilidades latentes y, al ser efectos fijos, pueden ser modelados como función de variables que permanecen constantes a lo largo de la vida del niño, que podrían determinar las habilidades latentes y sean comunes a ambas bases de datos. De esta manera, podremos predecir el valor de estas habilidades latentes en la muestra Enhab. Algunos controles que podrían ser adecuados para modelar dichos efectos fijos son el género del niño y el nivel educacional de los padres.

$$
\hat{L A} A_{i}=\gamma_{0}^{L A}+\gamma_{1}^{L A} Z_{i}+\mu_{i}^{L A}
$$

La segunda etapa propone partir de estos efectos estimados y aprovechar la información sobre las "características permanentes" para la 
muestra Enhab, con el fin de obtener valores predichos de las habilidades latentes para tal muestra. Dicho de otra forma, el valor predicho de las habilidades latentes para la muestra Enhab $\left(\hat{L}_{i}\right)$ será el resultado de las "características permanentes" observadas $\left(Z_{i}\right)$ y los efectos previamente estimados $\left(\gamma_{1}^{\hat{L} A}\right)$ :

$$
\hat{\hat{A}_{i}}=\hat{\gamma}_{0}^{L A}+\hat{\gamma}_{1}^{L A} Z_{i}
$$

Un supuesto importante en este proceso de "emparejamiento" es que las muestras del ENM y de la Enhab son representativas a nivel nacional (es decir, evidencian características similares) ${ }^{5}$. Así, con los valores predichos, es posible estimar una ecuación de salarios y, más aún, analizar las fuerzas detrás de la existencia de una brecha salarial de género. Para ello, modelaremos los salarios como función de las habilidades cognitivas y no cognitivas latentes identificadas a partir de la metodología econométrica descrita líneas arriba.

Con la muestra de Enhab que contiene información de salarios, medidas de habilidad y habilidades latentes aplicaremos dos aproximaciones para analizar el rol de las habilidades en los salarios. La primera consiste en aplicar el método de descomposición de Blinder-Oaxaca, el cual permite evaluar el rol de brechas en regresores sobre brechas en una variable de resultado. La segunda desarrolla el modelo completo de decisión del individuo, incorporando interacciones entre las habilidades y una variable dicotómica de género para analizar el rol de brechas de habilidad en brechas de salario. Así será posible enriquecer el análisis efectuando una comparación entre los resultados obtenidos haciendo uso de medidas de habilidad observables, por un lado, y (proxies de) habilidades latentes, por el otro. De esta manera, tanto las medidas de habilidad observables como las habilidades latentes nos servirán para evaluar el rol de las habilidades en la brecha salarial de género.

\subsection{Brecha salarial de género y el método de descomposición de Blinder-Oaxaca}

El método de descomposición de Blinder-Oaxaca es una metodología que permite descomponer diferencias en el salario promedio de dos grupos, en este caso, hombres y mujeres. Se parte de un modelo lineal

${ }^{5}$ Debemos tomar en cuenta que el ENM ignora la información de aquellos niños que pertenecen a hogares que perciben los ingresos más altos. 
(separable) que es explicado por características observables y no observables:

$$
Y_{g}=X \beta_{g}+\eta_{g} \text { parag-hombre,mujer }
$$

Donde, sea $g$ una variable dicotómica que indica pertenencia a un grupo determinado, $Y_{g}$ el resultado de interés para un miembro del grupo $g, X_{g}$ el vector de características observables (incluyendo una constante), $\beta_{\mathrm{g}}$ el vector columna de coeficientes de una regresión lineal de $Y_{g}$ sobre $X_{g}$, y las barras representan promedios. Se pueden reexpresar, así, las diferencias salariales como diferencias en características observables o diferencias en los coeficientes de dichas características:

$$
\left(\bar{Y}^{1}-\bar{Y}^{0}=\left(\bar{X}^{1}-\bar{X}^{0}\right) \hat{\beta}^{1}+\bar{X}^{0}\left(\hat{\beta}^{1}-\hat{\beta}^{0}\right)\right.
$$

donde el primer y segundo términos del lado derecho de la ecuación representan los componentes explicados y no explicados de la brecha en el resultado promedio, respectivamente. A esto se le denomina "descomposición doble", ya que precisamente descompone brechas entre diferencias en nivel o diferencias en retornos. Una extensión de esta metodología es la denominada "descomposición triple", la cual incluye un tercer componente que interactúa —y de manera simultánea- diferencias en niveles y retornos de los regresores considerados:

$$
\bar{Y}^{1}-\bar{Y}^{0}=\left(\bar{X}^{1}-\bar{X}^{0}\right) \hat{\beta}^{1}+\bar{X}^{0}\left(\hat{\beta}^{1}-\hat{\beta}^{0}\right)+\left(\bar{X}^{1}-\bar{X}^{0}\right)\left(\hat{\beta}^{1}-\hat{\beta}^{0}\right)
$$

en el cual el último término del lado derecho de la ecuación representa el término de interacción.

\section{RESULTADOS}

En esta sección comparamos los resultados de la estimación del efecto de las habilidades cognitivas y no cognitivas sobre los salarios obtenidos mediante el uso de puntajes de pruebas que miden dichas habilidades y aquellos resultados obtenidos luego de considerar las habilidades latentes estimadas. En cada caso, empezamos presentando los resultados obtenidos tras estimar una ecuación de Mincer del logaritmo 
de los salarios, controlando por el nivel educacional y las habilidades. Posteriormente, aplicamos el método de descomposición de BlinderOaxaca con el fin de estimar el impacto de las habilidades en la brecha salarial de género. Finalmente, en el afán de separar el efecto de las habilidades en la brecha salarial de género a través de cada una de las decisiones del individuo previas a la determinación del salario, estimamos un modelo conjunto de educación, empleo, elección de ocupación y salarios. Para ello, nos valemos del procedimiento, explicado previamente, para obtener estimados de las habilidades latentes cognitivas y no cognitivas y presentamos los resultados correspondientes a cada una de las cuatro etapas que lo conforman.

\subsection{Salarios y medidas de habilidad}

A propósito de la discusión previa acerca de los problemas asociados a la estimación del efecto de las habilidades en los salarios, la tabla 3 muestra los resultados de una ecuación de Mincer bajo el supuesto ingenuo- de que no existe correlación entre las medidas de habilidad y los años de educación. La columna 1 evidencia que luego de controlar por experiencia laboral, lugar de residencia y lengua materna, un año de educación adicional lleva a un incremento de 10,9 por ciento en los salarios. La columna 2 incorpora controles como el nivel educacional de los padres, ya que ello podría explicar parte de la correlación entre salarios y educación. Con esto, el coeficiente estimado cae de 10,9 a 9 por ciento. El incorporar medidas de habilidad cognitiva y no cognitiva pone en evidencia que las medidas de habilidad cognitiva y estabilidad emocional llevan a salarios más altos, mientras que la amabilidad y la consistencia en términos de esfuerzo parecen tener el efecto contrario. Asimismo, incluir dichas medidas de habilidad genera una reducción en el retorno a cada año de educación, lo cual sugiere que el coeficiente de la columna 2 estaría sobreestimando el efecto de la educación en los salarios.

\subsubsection{Método de descomposición de Blinder-Oaxaca}

Con el fin de estimar el efecto de las medidas de habilidad, optamos por aplicar el método de descomposición de Blinder-Oaxaca. En la muestra utilizada, el logaritmo del salario promedio es 1,417 para hom- 
Tabla 3. ECUACIÓN DE MINCER CON MEDIDAS DE HABILIDAD

\begin{tabular}{lrrr}
\hline & {$[1]$} & \multicolumn{1}{c}{$[2]$} & \multicolumn{1}{c}{$[3]$} \\
\hline Años de educación & $0.1091^{* * *}$ & $0.0934^{* * *}$ & $0.0804^{* * *}$ \\
Experiencia & 0.0255 & $0.0318^{*}$ & $0.0329^{*}$ \\
Experiencia ${ }^{2}$ & -0.0003 & -0.0004 & -0.0004 \\
Reside en Lima & 0.0397 & 0.0297 & 0.0091 \\
Lengua materna (español) & 0.2675 & 0.4296 & $0.5023^{* *}$ \\
Nivel educacional del padre & & 0.0148 & 0.0097 \\
Nivel educacional de la madre & & $0.0386^{* *}$ & $0.0366^{* *}$ \\
Goldberg, extroversión & & & 0.0257 \\
Goldberg, amabilidad & & & -0.0371 \\
Goldberg, cooperación & & & $-0.0616^{*}$ \\
Goldberg, actitud concienzuda (fuerte) & & & -0.005 \\
Goldberg, estabilidad emocional & & & $0.0810^{* * *}$ \\
Goldberg, apertura & & & -0.0069 \\
GRIT 2, consistencia de intereses & & & $-0.0453^{*}$ \\
GRIT 2, persistencia del esfuerzo & & & 0.0031 \\
Pruebas de habilidad cognitiva & & & $0.0751^{* * *}$ \\
Constante & & & $-0.8131^{* * *}$ \\
\hline No observaciones & & & 1079 \\
R-cuadrado & & & 0.187 \\
\hline
\end{tabular}

Nota: " indica un nivel de significancia del 10\%; ** indica un nivel de significancia del 5\%; $\mathrm{y}^{* \star *}$ indica un nivel de significancia del $1 \%$. La muestra corresponde a personas empleadas en el momento de la encuesta y con información disponible acerca de pruebas de habilidades y controles individuales.

bres y 1,141 para mujeres, llevando a una brecha salarial estadísticamente significativa de 0,276 . Sin embargo, nuestra atención se centra en la importancia relativa de los elementos que explican dicha brecha. Haciendo uso de los métodos de descomposición doble y triple descritos previamente, encontramos que los datos favorecen el hecho de que la brecha salarial es atribuible a diferencias entre los retornos y niveles de las habilidades. No obstante, si bien habría un incremento significativo en el salario por hora de las mujeres, más del 80 por ciento de la brecha salarial de género se reduciría si ellas compartieran el valor de los co- 
eficientes de los hombres, dadas sus propias características. La brecha salarial de género, así como el efecto de la dotación y las diferencias en los coeficientes son significativos, aun luego de controlar por características individuales comunes en este tipo de análisis. Las primeras dos columnas de la tabla 4 ilustran, luego de agregar diversos controles, los resultados obtenidos mediante la descomposición doble y el uso de una especificación sencilla, respectivamente. Las columnas 3 y 4 presentan los resultados obtenidos mediante la descomposición triple.

Un segundo ámbito de análisis corresponde a la contribución individual de los predictores al componente explicado de la brecha salarial de género. A partir de este momento, trabajaremos con la medida compuesta de GRIT como la prueba representativa que mide habilidades no cognitivas $^{6}$. La tabla 4 presenta la proporción de la brecha salarial en términos de regresores y coeficientes atribuidos a diferencias en medidas de habilidad cognitiva y no cognitiva. Las cuatro aproximaciones presentadas (columnas 1 a 4) evidencian que el "efecto de niveles" se debe, básicamente, a niveles heterogéneos de habilidades cognitivas (medidas) entre hombres y mujeres. En el caso de las habilidades no cognitivas, no se hallaron efectos significativos. Las brechas en términos de coeficientes (retornos) evidenciaron una estrecha relación con controles a nivel individual y heterogeneidad no observada entre géneros.

La descomposición de Blinder-Oaxaca puede ser interpretada como un modelo simple del ciclo de vida desde una perspectiva que considera factores latentes. Las habilidades cognitivas y no cognitivas determinan el nivel educacional. No obstante, recordemos que las habilidades latentes no son observables. Lo que se observa son medidas de estas habilidades. Por tanto, los retornos estimados mediante dicho método de descomposición son función de los retornos a la educación y los parámetros que gobiernan la elección del nivel educacional alcanzado por el individuo. Asimismo, es plausible suponer que los parámetros que influyen en la decisión de ocupación también estén afectando el retorno promedio, general, estimado.

${ }^{6}$ Se optó por trabajar con GRIT, ya que la literatura en torno al rol de las habilidades no cognitivas en el salario resalta su importancia. Asimismo, Díaz et al. (2012), autores que utilizaron también la Enhab, hallan evidencia a favor de su rol fundamental en ecuaciones de salario. No obstante, todas las estimaciones llevadas a cabo a lo largo del estudio se han realizado con las demás medidas de habilidad y se han obtenido resultados similares. 
Tabla 4. DESCOMPOSICIÓN DE BLINDER-OAXACA CON MEDIDAS DE HABILIDAD

\begin{tabular}{|c|c|c|c|c|}
\hline & [1] & [२] & [3] & [4] \\
\hline \multirow[t]{2}{*}{ Brecha salarial } & $0.276^{* * *}$ & $0.276^{* * *}$ & $0.276^{* * *}$ & $0.276^{* * *}$ \\
\hline & $(0.065)$ & $(0.051)$ & $(0.065)$ & $(0.067)$ \\
\hline \multirow[t]{2}{*}{ Niveles } & $0.059^{* * *}$ & $0.045^{* * *}$ & $0.067^{* * *}$ & $0.058^{* *}$ \\
\hline & (0.019) & (0.019) & $(0.024)$ & $(0.028)$ \\
\hline \multirow[t]{3}{*}{ Retornos } & $0.217^{* * *}$ & $0.231^{* \star *}$ & 0.225 & $0.242^{* * *}$ \\
\hline & $(0.063)$ & $(0.048)$ & $(0.067)$ & (0.062) \\
\hline & \multicolumn{4}{|c|}{ Niveles } \\
\hline \multirow[t]{2}{*}{ Cognitiva } & $0.059^{* * *}$ & $0.058^{* * *}$ & $0.067^{* * *}$ & $0.067^{* * *}$ \\
\hline & $(0.019)$ & $(0.015)$ & (0.023) & $(0.023)$ \\
\hline \multirow[t]{3}{*}{ No cognitiva } & 0.000 & 0.000 & 0.000 & 0.000 \\
\hline & (0.002) & $(0.001)$ & (0.002) & $(0.001)$ \\
\hline & \multicolumn{4}{|c|}{ Retornos } \\
\hline Cognitiva & $\begin{array}{r}-0.171 \\
(0.167)\end{array}$ & $\begin{array}{l}-0.192 \\
(0.130)\end{array}$ & $\begin{array}{l}-0.163 \\
(0.160)\end{array}$ & $\begin{array}{l}-0.184 \\
(0.144)\end{array}$ \\
\hline \multirow[t]{2}{*}{ No cognitiva } & 0.007 & -0.017 & 0.007 & -0.017 \\
\hline & $(0.143)$ & $(0.165)$ & $(0.144)$ & $(0.154)$ \\
\hline $\mathrm{N}^{\circ}$ observaciones & 1081 & 1081 & 1081 & 1081 \\
\hline Controles & No & Si & No & $\mathrm{Si}$ \\
\hline Interacciones & No & No & Sí & Sí \\
\hline
\end{tabular}

Nota: Errores estándar entre paréntesis se encuentran agrupados a nivel regional. * indica un nivel de significancia del $10 \%$; ${ }^{* *}$ indica un nivel de significancia del $5 \%$; ${ }^{* * *}$ indica un nivel de significancia del $1 \%$. La muestra corresponde a personas empleadas en el momento de la encuesta y con información disponible acerca de pruebas de habilidades y controles individuales. Los controles considerados son edad, edad al cuadrado y si el individuo reside en Lima.

\subsubsection{Estimación conjunta: educación, empleo, ocupación y salarios}

Los resultados obtenidos previamente brindan información acerca del rol de las medidas de habilidad cognitiva y no cognitiva sobre los salarios, pero no consideran las decisiones intermedias tomadas por los individuos antes de recibir un nivel de salario determinado. Con el fin de separar los efectos de las medidas de habilidad sobre cada una de estas decisiones, procedemos a estimar un modelo conjunto que considera elecciones de educación, empleo y ocupación. El mismo es consistente con la serie de decisiones que debe tomar un individuo a lo largo de su 
vida profesional. Tomando en cuenta su nivel de habilidades, un individuo elige cuál es el máximo nivel educacional que alcanza; luego, dado el nivel educacional que posee y sus habilidades latentes, decide participar o no del mercado laboral. Una vez que decide ser empleado, debe elegir su ocupación y, finalmente, recibir un salario acorde con sus decisiones previas y su nivel de habilidades. Para llevar a cabo un análisis de este tipo, consideramos dos niveles educacionales (secundaria completa es el punto de corte), la condición de ser empleado o no serlo, y dos clases de ocupación: "white collar" (ocupaciones que requieren capacitación especializada) y "blue collar" (ocupaciones que no requieren capacitación especializada).

Los resultados de la estimación por máxima verosimilitud del modelo conjunto se presentan en la tabla 5. El procedimiento de estimación requiere maximizar la verosimilitud conjunta $(L)$ de adquirir un determinado nivel educacional $(s)$, estar empleado $\left(\mathrm{h}_{\mathrm{i}}=1\right)$, haber elegido una ocupación que requiera capacitación especializada $\left(\mathrm{o}_{\mathrm{i}}=1\right)$ y ganar un cierto nivel de salarios $(w)$. Por tanto, la contribución individual a la verosimilitud sería:

$l_{i}=\underbrace{L_{s i}\left(\theta_{S} \mid L A_{i}\right)}_{\text {Educación }} \overbrace{L_{h i}\left(\theta_{h} \mid L A_{i}, s_{i}\right)}^{\text {Empleo }} \underbrace{L_{o i}\left(\theta_{O} \mid L A_{i}, s_{i}, h_{i}=1\right)}_{\text {Ocupacin }} \overbrace{L_{w i}\left(\theta_{W} \mid L A_{i}, s_{i}, h_{i}=1, o_{i}\right)}^{\text {Salarios }}$

Cada columna de la tabla 5 corresponde a cada una de las decisiones involucradas en el modelo. En el modelo probabilístico de selección de educación controlamos por el nivel educacional de los padres. En el caso de la decisión de trabajo, controlamos por el nivel de desempleo promedio de la región a la que pertenece y una variable dicotómica que identifica la región en la que reside el niño. En tanto, para la decisión de ocupación se controla por edad. Los resultados indican que mientras las medidas de habilidad cognitiva parecen importar más en la determinación de los años de educación y la elección de ocupación, las medidas de habilidad no cognitiva son más importantes para la determinación de los salarios y la empleabilidad. En términos de diferencias de género, los hombres parecen tener un mayor retorno que las mujeres en general, pero en particular para el caso de habilidades no cognitivas en términos 
de empleabilidad y mayores salarios. Las mujeres obtienen mayores retornos a las habilidades cognitivas sólo tratándose de la elección del nivel educacional. No obstante, debemos considerar que los impactos estimados consideran medidas de habilidad observables, lo cual podría estar capturando el efecto de otros factores correlacionados con las variables dependientes y estas medidas de habilidad.

Tabla 5. ESTIMACIÓN CONJUNTA CON MEDIDAS DE HABILIDAD

\begin{tabular}{lcccc}
\hline Modelos & Educación & Empleo & Ocupación & Salarios por hora \\
\hline Cognitiva (mujeres) & $0.046^{\star * *}$ & $-0.004^{*}$ & $0.043^{* * *}$ & -0.030 \\
& $(0.003)$ & $(0.002)$ & $(0.004)$ & $(0.018)$ \\
Interacción c/Cognitiva & $-0.006^{\star \star *}$ & 0.004 & -0.007 & $0.040^{\star}$ \\
& $(0.003)$ & $(0.003)$ & $(0.005)$ & $(0.022)$ \\
No cognitiva (mujeres) & $0.190^{* \star *}$ & $0.122^{\star *}$ & 0.129 & $0.996^{* *}$ \\
& $(0.067)$ & $(0.057)$ & $(0.109)$ & $(0.467)$ \\
Interacción c/No cognitiva & 0.134 & $0.195^{* *}$ & 0.105 & $1.335^{* *}$ \\
& $(0.093)$ & $(0.076)$ & $(0.134)$ & $(0.605)$ \\
\hline
\end{tabular}

$\mathrm{N}^{\circ}$ observaciones

2421

Nota: ${ }^{*}$ indica un nivel de significancia del $10 \%$; ${ }^{*}$ indica un nivel de significancia del $5 \%$; y *** indica un nivel de significancia del $1 \%$. El término de interacción equivale al producto: (Hombre)*(Medida de habilidad).

\subsection{Salarios y habilidades latentes}

Con el objetivo de estimar el efecto neto de las habilidades sobre la brecha salarial de género, debemos considerar las habilidades latentes. A continuación presentamos los resultados del procedimiento propuesto para estimar dichas habilidades latentes (cognitivas y no cognitivas) y, luego, reestimamos los modelos introducidos previamente, considerando estas habilidades.

\subsubsection{Aproximando habilidades latentes}

La tabla 6 presenta los resultados de la primera etapa. Las columnas 1 a 3 corresponden a las estimaciones de las medidas de autoestima, autoeficacia y habilidad cognitiva (puntaje de la prueba TVIP), 
Tabla 6. ESTIMACIÓN DE LA PRIMERA ETAPA (MODELO DE EFECTOS FIJOS MEDIDAS DE HABILIDAD)

\begin{tabular}{|c|c|c|c|}
\hline & Autoestima & Autoeficacia & $\begin{array}{l}\text { Habilidad } \\
\text { cognitiva }\end{array}$ \\
\hline \multirow[t]{2}{*}{ Habilidad del cuidador principal } & $0.039^{\star * *}$ & $0.099^{* * *}$ & - \\
\hline & $(0.013)$ & $(0.023)$ & - \\
\hline \multirow[t]{2}{*}{ Índice de riqueza } & 0.027 & 0.248 & -2.793 \\
\hline & $(0.180)$ & $(0.261)$ & $(1.854)$ \\
\hline Talla para la edad (estandarizado) & $\begin{array}{l}-0.136^{* * *} \\
(0.047)\end{array}$ & $\begin{array}{l}0.187^{\star \star \star} \\
(0.038)\end{array}$ & $\begin{array}{l}-1.698^{* \star \star} \\
(0.587)\end{array}$ \\
\hline \multirow{2}{*}{$\begin{array}{l}\text { Índice de masa corporal } \\
\text { (estandarizado) }\end{array}$} & $0.068^{*}$ & 0.097 & $-1.377^{\star * *}$ \\
\hline & $(0.023)$ & $(0.075)$ & $(0.302)$ \\
\hline \multirow[t]{2}{*}{ Edad (en meses) } & -0.002 & 0.001 & $0.793^{* * *}$ \\
\hline & $(0.001)$ & $(0.001)$ & $(0.013)$ \\
\hline \multirow[t]{2}{*}{ Estatus socioeconómico (percibido) } & $0.343^{* * *}$ & 0.007 & $1.144^{\star \star \star}$ \\
\hline & $(0.035)$ & $(0.039)$ & $(0.376)$ \\
\hline $\begin{array}{l}\text { Ausencia a la escuela por problemas } \\
\text { de salud }\end{array}$ & $\begin{array}{l}-0.140^{* * *} \\
(0.048)\end{array}$ & $\begin{array}{l}-0.271^{* * \star} \\
(0.049)\end{array}$ & $\begin{array}{c}-1.416^{* \star *} \\
(0.462)\end{array}$ \\
\hline \multirow[t]{2}{*}{ Logaritmo del consumo del hogar } & 0.087 & -0.043 & -0.363 \\
\hline & $(0.060)$ & (0.062) & $(0.553)$ \\
\hline$N^{\circ}$ observaciones & 349 & 349 & 349 \\
\hline R-cuadrado & 0.035 & 0.025 & 0.820 \\
\hline
\end{tabular}

Nota: Se considera la muestra de niños que viven en zonas urbanas y tienen información sobre todas las variables incluidas en la regresión. * indica un nivel de significancia del $10 \% ;{ }^{* *}$ indica un nivel de significancia del $5 \%$; ${ }^{* * *}$ indica un nivel de significancia del $1 \%$ de la prueba de diferencia de medias entre hombres y mujeres. Errores estándares agrupados a nivel de comunidad.

respectivamente. Cada regresión controla por la medida de habilidad correspondiente del cuidador principal del niño ${ }^{7}$, la medida estandarizada de la talla para la edad del niño, el índice de masa corporal del niño, su edad, la percepción del estatus socioeconómico del hogar, un índice de riqueza del hogar, una variable que indica si el niño se ausentó a la escuela por problemas de salud durante más de una semana (variación en la educación) y el logaritmo del consumo real per cápita. Los errores estándar se encuentran agrupados por comunidad.

${ }^{7}$ La base de datos no contenía medida de habilidad cognitiva para el cuidador principal de los niños. 
Cambios en la medida de autoestima del cuidador principal, la talla para la edad, el índice de masa corporal, haber faltado a la escuela por problemas de salud y el estatus socioeconómico percibido evidenciaron una relación estadísticamente significativa con la medida de autoestima del niño, siendo el último factor el más importante. Los resultados son similares para el caso de la medida de autoeficacia, pero cambios en el estatus socioeconómico percibido no son estadísticamente significativos. En cuanto a la medida de habilidad cognitiva, la variación en todos los controles incluidos fue estadísticamente significativa con excepción del índice de riqueza del hogar. La talla para la edad, el índice de masa corporal y el haber faltado a la escuela por problemas de salud evidenciaron un efecto negativo sobre el puntaje de la prueba.

La tabla 7 presenta los resultados de la segunda etapa del procedimiento propuesto. Los controles considerados son el género y el nivel educacional de los padres. La literatura en torno a la formación de habilidades sugiere que la habilidad "latente" corresponde a la habilidad innata y, por tanto, debería ser afectada por variables determinadas para el niño sólo hasta los tres años de vida. En el presente estudio, lo que estimamos como habilidad latente en realidad es la habilidad formada hasta los 12 años del niño, por lo que es de suponer que las variables fijas hasta dicho momento serían importantes para determinar el nivel de habilidades latentes del niño. Controles como el nivel educacional de los padres, el género del niño y la lengua materna son algunas de estas características importantes, pero no las características asociadas a la educación secundaria recibida (por problemas de endogeneidad). Este hecho es el que motiva la forma reducida que desarrollamos. En el caso de las tres proxies de habilidad latente, todos los controles incluidos ${ }^{8} \mathrm{se}$ mostraron estadísticamente significativos. Mientras las mujeres evidencian un mayor nivel de autoeficacia, lo opuesto ocurre para las habilidades cognitivas. Asimismo, el nivel educacional de los padres tiene un impacto positivo en todas las habilidades latentes predichas ${ }^{9}$.

${ }^{8}$ La variable de lengua materna no fue considerada para el análisis. Esto, porque alrededor del 90 por ciento de la muestra tiene el español como lengua materna, lo que implica poca variabilidad en dicha característica.

9 En general, los resultados obtenidos para dichas estimaciones presentan niveles bajos de ajuste, lo cual podría atribuirle un rol importante a variables no observables. Se llevaron a cabo estimaciones considerando otras posibles variables relevantes disponibles, pero no se observaron incrementos relevantes en el nivel de 
Tabla 7. ESTIMACIÓN DE LA SEGUNDA ETAPA (HABILIDADES LATENTES SOBRE CARACTERÍSTICAS PERMANENTES, ENM)

\begin{tabular}{|c|c|c|c|}
\hline & Autoestima & Autoeficacia & $\begin{array}{l}\text { Habilidad } \\
\text { cognitiva }\end{array}$ \\
\hline \multirow[t]{2}{*}{ Género (hombre) } & 0.012 & $-0.361^{* \star *}$ & $1.026^{* \star *}$ \\
\hline & $(0.016)$ & $(0.013)$ & $(0.361)$ \\
\hline \multirow[t]{2}{*}{ Nivel educacional del padre } & $0.035^{\star \star *}$ & $0.018^{* * \star}$ & $1.442^{* * *}$ \\
\hline & $(0.006)$ & $(0.004)$ & $(0.075)$ \\
\hline Nivel educacional de la madre & $\begin{array}{l}0.016^{\star * *} \\
(0.002)\end{array}$ & $\begin{array}{c}-0.008^{\star *} \\
(0.003)\end{array}$ & $\begin{array}{l}0.461^{\star \star \star} \\
(0.090)\end{array}$ \\
\hline Constante & $\begin{array}{c}-0.581^{* * *} \\
(0.071)\end{array}$ & $\begin{array}{l}0.43^{\star \star \star} \\
(0.044)\end{array}$ & $\begin{array}{c}-61.802^{* * \star} \\
(1.326)\end{array}$ \\
\hline $\mathrm{N}^{\circ}$ observaciones & 349 & 349 & 349 \\
\hline R-cuadrado & 0.048 & 0.067 & 0.169 \\
\hline
\end{tabular}

Nota: * indica un nivel de significancia del $10 \%$; ${ }^{* \star}$ indica un nivel de significancia del $5 \%$; $\mathrm{y}^{* * *}$ indica un nivel de significancia del $1 \%$ de la prueba de diferencia de medias entre hombres y mujeres. Errores estándares agrupados a nivel de comunidad.

La tabla 8 muestra estadísticas descriptivas de las predicciones realizadas en ambas bases de datos (Niños del Milenio y Enhab) para la muestra completa y la brecha de género. Como se puede observar, ambas predicciones evidencian patrones similares en términos de la dirección o signo de las brechas.

Finalmente, se muestran los resultados de la estimación del modelo de salarios en función de las habilidades latentes. La tabla 9 compara los resultados obtenidos tras estimar una ecuación de Mincer con medidas de habilidad (columna 3 ) y con habilidades latentes (columna 4) ${ }^{10}$. Vale la pena resaltar dos resultados. En primer lugar, el retorno a la edu-

ajuste (e.g. la presencia del padre en los primeros años del niño, el gasto per cápita no asociado a comestibles y la presencia de un evento que pudiera haber afectado el bienestar del hogar desde el nacimiento del niño). Debido a limitaciones de información, no es posible controlar por otras variables que pudieran haberse mantenido fijas hasta los 12 años, por lo que esto representaría una limitación para nuestra aproximación. Algunas explicaciones posibles para esta productividad diferenciada entre hombres y mujeres asociadas a los factores fijos en los primeros años del niño podrían ser de naturaleza más cultural (i.e. en el mundo rural se suele priorizar que el hijo varón asista a la escuela en perjuicio de las hijas, a quienes se les atribuye un rol asociado a las labores domésticas y al cuidado de los hermanos menores).

${ }^{10}$ A partir de este momento utilizaremos únicamente la autoeficacia como la habilidad no cognitiva representativa. 
Tabla 8. ESTADÍSTICAS DE LA TERCERA ETAPA (HABILIDADES LATENTES PREDICHAS EN AMBAS BASES DE DATOS)

\begin{tabular}{|c|c|c|c|c|}
\hline Niños del Milenio & Mujeres & Hombres & $\begin{array}{c}\text { Brecha de } \\
\text { género }\end{array}$ & $\begin{array}{l}\text { Muestra } \\
\text { completo }\end{array}$ \\
\hline Habilidad cognitiva latente & -42.676 & -40.854 & $\begin{array}{c}1.821 \\
(1.462)\end{array}$ & -41.716 \\
\hline Autoeficacia latente & 0.532 & 0.182 & $\begin{array}{c}-0.350^{* * *} \\
(0.073)\end{array}$ & 0.347 \\
\hline Autoestima latente & -0.067 & -0.036 & $\begin{array}{c}0.031 \\
(0.074)\end{array}$ & -0.050 \\
\hline $\mathrm{N}^{\circ}$ observaciones & 165 & 184 & 349 & \\
\hline Enhab & Mujeres & Hombres & $\begin{array}{c}\text { Brecha de } \\
\text { género }\end{array}$ & $\begin{array}{l}\text { Muestra } \\
\text { completo }\end{array}$ \\
\hline Habilidad cognitiva latente & -47.409 & -46.209 & $\begin{array}{l}1.201^{* * *} \\
(0.201)\end{array}$ & -46.829 \\
\hline Autoeficacia latente & 0.516 & 0.156 & $\begin{array}{c}-0.361^{\star * *} \\
(0.001)\end{array}$ & 0.342 \\
\hline Autoestima latente & -0.198 & -0.193 & $\begin{array}{c}0.005 \\
(0.005)\end{array}$ & -0.196 \\
\hline
\end{tabular}

$\mathrm{N}^{\circ}$ observaciones

3872

3627

7499

Nota: Los efectos fijos predichos para la muestra de Enhab fueron construidos sobre la base de los estimados de la muestra urbana de Niños del Milenio. Errores estándares a justados ante la presencia de correlación intragrupos se reportan entre paréntesis. * indica un nivel de significancia del $10 \%$; ${ }^{* *}$ indica un nivel de significancia del $5 \%$; ${ }^{* * *}$ indica un nivel de significancia del $1 \%$.

cación, considerando la especificación de la columna 4, es mayor que el correspondiente a la columna 3 y más similar al de la columna 2. Este hecho es consistente con la hipótesis de que las medidas de habilidad capturan parte del efecto de la educación sobre el salario (lo cual explicaría la caída en el retorno entre las columnas 2 y 3). En segundo lugar, cabe resaltar el cambio en la significancia estadística de las habilidades no cognitivas. Ello pone en evidencia que existe una relación entre habilidades no cognitivas y salarios, pero también implica que podemos considerar el efecto indirecto de las habilidades a través del nivel educacional al poder controlar por el nivel educacional y las habilidades latentes (netas). 
Tabla 9. ECUACIÓN DE MINCER CON HABILIDADES LATENTES

\begin{tabular}{|c|c|c|c|c|}
\hline & [1] & [2] & [3] & [4] \\
\hline Años de educación & $0.1091^{* \star *}$ & $0.0934^{* * *}$ & $0.0804^{* * *}$ & $0.0914^{* * *}$ \\
\hline Experiencia & 0.0255 & $0.0318^{*}$ & $0.0329^{*}$ & $0.0333^{* *}$ \\
\hline Experiencia $^{2}$ & -0.0003 & -0.0004 & -0.0004 & -0.0004 \\
\hline Reside en Lima & 0.0397 & 0.0297 & 0.0091 & $0.0606^{*}$ \\
\hline Lengua materna (español) & 0.2675 & 0.4296 & $0.5023^{* *}$ & 0.2975 \\
\hline Nivel educacional del padre & & 0.0148 & 0.0097 & 0.0507 \\
\hline Nivel educacional de la madre & & $0.0386^{* *}$ & $0.0365^{* *}$ & 0.0312 \\
\hline Goldberg, extroversión & & & 0.0257 & \\
\hline Goldberg, amabilidad & & & -0.0371 & \\
\hline Goldberg, cooperación & & & $-0.0616^{*}$ & \\
\hline $\begin{array}{l}\text { Goldberg, actitud concienzuda } \\
\text { (fuerte) }\end{array}$ & & & -0.005 & \\
\hline Goldberg, estabilidad emocional & & & $0.810^{\star \star *}$ & \\
\hline Goldberg, apertura & & & -0.0069 & \\
\hline GRIT 2, consistencia de interes & & & $-0453^{*}$ & \\
\hline GRIT 2, persistencia del esfuerzo & & & 0.0031 & \\
\hline Pruebas de habilidad cognitiva & & & $0.0751^{\star \star *}$ & \\
\hline $\begin{array}{l}\text { Habilidad no cognitiva latente } \\
\text { (predicha) }\end{array}$ & & & & $-0.7259^{* * *}$ \\
\hline $\begin{array}{l}\text { Habilidad cognitiva latente } \\
\text { (predicha) }\end{array}$ & & & & $-0.7259^{* * *}$ \\
\hline Constante & $-06028^{\star *}$ & $-0.9125^{*}+$ & $-0.8131^{* * *}$ & -0.8876 \\
\hline $\mathrm{N}^{\circ}$ observaciones & 1079 & 1079 & 1073 & 1079 \\
\hline R-cuadrado & 0.157 & 0.17 & 0.187 & 0.193 \\
\hline
\end{tabular}

Nota: Errores estándar robustos entre paréntesis se encuentran agrupados a nivel regional. " indica un nivel de significancia del 10\%; ${ }^{* *}$ indica un nivel de significancia del 5\%; $y^{* * *}$ indica un nivel de significancia del $1 \%$. La muestra corresponde a personas empleadas en el momento de la encuesta y con información disponible acerca de pruebas de habilidades y controles individuales. 


\subsubsection{Método de descomposición de Blinder-Oaxaca}

La presente sección describe los resultados obtenidos tras aplicar el método de descomposición de Blinder-Oaxaca a la muestra completa en edad de trabajar ${ }^{11}$, pero considerando las diferencias en habilidades latentes ya predichas. Como se planteó previamente para el caso en que utilizamos medidas de habilidad, existe una brecha salarial de género significativa. A diferencia de los resultados obtenidos en la sección anterior, la brecha hallada por medio de la nueva especificación no es atribuible sólo a diferencias en retornos o niveles de habilidades cognitivas, sino que las habilidades no cognitivas también parecen importantes para explicar la brecha salarial de género.

La tabla 10 muestra los resultados de la descomposición de Blinder-Oaxaca para la muestra de la Enhab considerando brechas en habilidades cognitivas latentes así como en autoeficacia (latente). Luego de aplicar las descomposiciones "doble" y "triple", los datos muestran evidencia a favor del hecho de que la brecha salarial de género es atribuible a diferencias en los coeficientes y predictores. En cuanto a diferencias en los retornos, es posible identificar que los hombres gozan de un mayor retorno a las habilidades cognitivas, lo cual genera un incremento en la brecha salarial de género, pero no existen diferencias estadísticas en los retornos a las habilidades no cognitivas que afecten la brecha. En cuanto a las diferencias en los niveles de las habilidades, la tabla 10 refleja qué diferencias en las habilidades cognitivas y no cognitivas favorecen a los hombres en términos de salario. Si bien el mayor acervo de habilidades cognitivas entre los hombres contribuye a incrementar la brecha salarial de género, la evidencia muestra que si las mujeres tuvieran el mismo nivel de habilidades no cognitivas que los hombres ganarían salarios significativamente mayores ${ }^{12}$. A primera vis-

${ }^{11}$ Cabe resaltar que el tamaño de la muestra en este caso es mayor al caso en que se aplicó Oaxaca-Blinder con medidas de habilidad, ya que se considera también a aquellos individuos sin información para medidas de habilidad. Procedemos de esta manera con el fin de aprovechar al máximo la variabilidad de los datos disponibles.

${ }^{12}$ A fin de identificar el efecto real de las habilidades no cognitivas en los salarios, se estimó una regresión de las habilidades no cognitivas en función de variables que representaran inversión por parte de los padres —inversión a una edad temprana, tales como si el niño vivía con el padre o lo había visto hace menos de seis meses, si el hogar había sido víctima de algún choque externo que afectara el bienestar del mismo, etc.- , tras lo cual no se obtuvieron resultados robustos que respaldaran esta teoría. 
ta, ello parece contradecir lo observado mediante los estadísticos descriptivos calculados inicialmente, en el que las mujeres mostraban mayores niveles de autoeficacia. No obstante, este fenómeno se explica por el hecho de que el retorno a las habilidades no cognitivas de las mujeres es negativo (como se hubiera podido observar si hubiéramos estimado la ecuación de Mincer con habilidades latentes por género). Por tanto, un mayor acervo de una habilidad que goza de un retorno negativo en el mercado genera, en efecto, un impacto negativo, perjudicando a las mujeres e incrementando la brecha salarial de género.

Tabla 10. DESCOMPOSICIÓN DE BLINDER-OAXACA CON HABILIDADES LATENTES

\begin{tabular}{|c|c|c|c|c|}
\hline & [1] & [2] & [3] & [4] \\
\hline \multirow[t]{2}{*}{ Brecha salarial } & $0.241^{\star * *}$ & $0.241^{\star \star \star}$ & $0.241^{\star * *}$ & $0.241^{\star \star \star}$ \\
\hline & (0.032) & $(0.027)$ & (0.032) & $(0.035)$ \\
\hline \multirow[t]{2}{*}{ Niveles } & $0.419^{* * *}$ & $0.607^{\star * *}$ & $0.458^{* * *}$ & $0.618^{* * *}$ \\
\hline & $(0.117)$ & $(0.134)$ & $(0.148)$ & $(0.152)$ \\
\hline \multirow[t]{2}{*}{ Retornos } & $\begin{array}{l}-0.178 \\
(0.124)\end{array}$ & $\begin{array}{c}-0.366^{* * *} \\
(0.136)\end{array}$ & $\begin{array}{l}-0.160 \\
(0.157)\end{array}$ & $\begin{array}{c}-0.367^{* * *} \\
(0.141)\end{array}$ \\
\hline & \multicolumn{4}{|c|}{ Niveles } \\
\hline \multirow[t]{2}{*}{ Cognitiva } & $0.026^{* * *}$ & $0.037^{\star \star *}$ & $0.034^{* * *}$ & $0.043^{* * *}$ \\
\hline & $(0.008)$ & $(0.010)$ & $(0.011)$ & $(0.013)$ \\
\hline \multirow[t]{3}{*}{ No cognitiva } & $0.393^{* * *}$ & $0.580^{* * \star}$ & $0.424^{\star \star *}$ & $0.585^{* * *}$ \\
\hline & $(0.115)$ & (0.132) & $(0.144)$ & $(0.148)$ \\
\hline & \multicolumn{4}{|c|}{ Retornos } \\
\hline \multirow[t]{2}{*}{ Cognitiva } & $0.540^{* * *}$ & $0.477^{*}$ & $0.544^{* * *}$ & $0.481^{* *}$ \\
\hline & $(0.178)$ & $(0.254)$ & $(0.180)$ & $(0.187)$ \\
\hline \multirow[t]{2}{*}{ No cognitiva } & 0.049 & 0.004 & 0.063 & -0.001 \\
\hline & $(0.186)$ & (0.272) & $(0.265)$ & $(0.270)$ \\
\hline $\mathrm{N}^{\circ}$ observaciones & 4079 & 4079 & 4079 & 4079 \\
\hline Controles & No & Si & No & Si \\
\hline Interacciones & No & No & Si & Si \\
\hline
\end{tabular}

Nota: Errores estándar entre paréntesis se encuentran agrupados a nivel regional. " indica un nivel de significancia del 10\%; ** indica un nivel de significancia del $5 \%$; $\mathrm{y}^{* \star *}$ indica un nivel de significancia del $1 \%$. La muestra corresponde a personas empleadas en el momento de la encuesta y con información disponible acerca de pruebas de habilidades y controles individuales. Los controles considerando son edad, edad al cuadrado y si el individuo reside en Lima. 


\subsubsection{Estimación conjunta: educación, empleo, ocupación y salarios}

La tabla 11 muestra los resultados de la estimación conjunta considerando las habilidades latentes cognitivas y no cognitivas. El considerar el rol de ambos tipos de habilidad en los procesos de elección considerados conlleva a resultados interesantes. En primer lugar, la habilidad cognitiva latente se muestra fundamental para lograr mayores niveles de educación, en similar magnitud para hombres y mujeres. Las habilidades no cognitivas parecen irrelevantes para esta primera decisión. Los siguientes tres procesos de elección deben ser interpretados de manera conjunta. En términos generales, es posible identificar que hay diferencias de género importantes en los retornos tratándose de las habilidades cognitivas y no cognitivas; ello en cada una de las tres variables de resultado analizadas. Lo anterior se encuentra explicado por lo importante que resulta considerar la dinámica detrás de la elección de la ocupación y por el hecho de que las mujeres tienen un mayor nivel de habilidades no cognitivas, las cuales, a su vez, cuentan con un retorno negativo. A propósito de ello, es posible interpretar que los hombres ganan mayores salarios por haber sido empleados en ocupaciones que premian más las habilidades cognitivas. Ello, junto con el hecho de que los hombres gozan de un mayor nivel de este tipo de habilidades, ayuda a explicar la existencia de una brecha salarial de género que los favorece. Asimismo, el análisis conjunto pone en evidencia que el retorno a las habilidades no cognitivas, en términos de empleo y salarios, es mayor para los hombres, aun cuando éstos presentan un menor acervo de este tipo de habilidades.

En contraste con los resultados de la estimación conjunta utilizando medidas de habilidad observables, es posible identificar que las diferencias a favor de los hombres en habilidades cognitivas y no cognitivas se reducen cuando se consideran las habilidades latentes. Incluso en el caso de las habilidades cognitivas latentes, las brechas se tornan significativas a favor de las mujeres. Más aún, el retorno a ambos tipos de habilidad latente gana significancia estadística en la ecuación de la decisión de la ocupación. Esta evidencia favorece la hipótesis de que gran parte de las diferencias atribuibles a brechas de habilidad ocurren en la dinámica que gobierna la elección de ocupaciones. 
Tabla 11. ESTIMACIÓN CONJUNTA CON HABILIDADES LATENTES

\begin{tabular}{|c|c|c|c|c|}
\hline Modelos & Educación & Empleo & Ocupación & Salarios por hora \\
\hline \multirow[t]{2}{*}{ Cognitiva (mujeres) } & $0.055^{\star * *}$ & $-0.014^{\star * \star}$ & $0.065^{\star * *}$ & $-0.076^{* * *}$ \\
\hline & $(0.004)$ & $(0.002)$ & $(0.004)$ & $(0.018)$ \\
\hline \multirow[t]{2}{*}{ Interacción c/Cognitiva } & -0.005 & $-0.009^{* * *}$ & $-0.014^{\star * *}$ & $-0.055^{\star * *}$ \\
\hline & $(0.003)$ & $(0.002)$ & $(0.004)$ & $(0.017)$ \\
\hline \multirow[t]{2}{*}{ No cognitiva (mujeres) } & 0.109 & -0.117 & $-1.539^{\star * \star}$ & -3.013 \\
\hline & $(0.443)$ & $(0.298)$ & $(0.483)$ & (2.314) \\
\hline \multirow[t]{2}{*}{ Interacción c/No cognitiva } & -0.515 & $0.817^{* *}$ & $-1.347^{* * *}$ & $5.794^{\star *}$ \\
\hline & $(0.438)$ & $(0.319)$ & $(0.512)$ & (2.463) \\
\hline
\end{tabular}

$\mathrm{N}^{\circ}$ observaciones 7499

Nota: ${ }^{*}$ indica un nivel de significancia del 10\%; ${ }^{* *}$ indica un nivel de significancia del 5\%; $\mathrm{y}^{* * *}$ indica un nivel de significancia del $1 \%$. El término de interacción equivale al producto: (Hombre)*(Habilidad latente).

\section{CONCLUSIONES}

El procedimiento econométrico y el modelo desarrollados en este documento evidencian algunos hechos importantes. En primer lugar, se confirma la existencia de una brecha salarial de género estadísticamente significativa para el Perú. Más aún, las habilidades cognitivas y no cognitivas juegan un rol importante en la formación de dicha brecha.

Los análisis comparativos, que utilizan medidas de habilidad observables y los estimados de las habilidades latentes, van en línea con la preocupación teórica respecto a problemas de endogeneidad. Asimismo, si bien al utilizar las medidas de habilidad, las no cognitivas no parecían importar para explicar las brechas salariales de género, lo opuesto se evidencia al utilizar las habilidades latentes estimadas. En efecto, el considerar un modelo conjunto de educación, empleo, ocupación y salarios reveló que ambos tipos de habilidad latente son importantes no solo en la determinación del salario, sino que son aún más importantes en las decisiones de educación y ocupación del individuo. Si bien las mujeres reportan menores salarios que los hombres a pesar de tener mayores niveles de habilidad no cognitiva, esto parece estar asociado al hecho de que dichas habilidades son valoradas negativamente en el mercado 
laboral. Al mismo tiempo, los hombres tienen un mayor nivel de habilidades que brindan un premio en términos de variables del mercado laboral.

\section{ALGUNAS IMPLICANCIAS DE POLÍTICAS PÚBLICAS}

Este artículo se inscribe en la creciente literatura teórica y empírica, de América Latina (Bassi y Galiani 2009; Urzúa et al. 2009) y el Perú (Díaz et al. 2012; Castro y Yamada 2012), que resalta el importante papel que desempeña la formación de habilidades cognitivas y no cognitivas a lo largo del ciclo de vida de los individuos, tanto para la progresión y éxito en el sistema educativo, como para la participación y resultados posteriores en el mercado laboral.

Estudios previos sobre diferencias de género en el Perú (véase, por ejemplo, Felices 1996; Ñopo 2009) han estimado una brecha salarial de género no explicada (es decir, luego de controlar por distintos stocks de escolaridad, experiencia laboral y otras variables sociodemográficas) que fluctúa entre 15 y 28 por ciento. Los resultados del presente estudio nos indican que una parte de esa brecha no explicada se debería a las diferencias en dotaciones de habilidades cognitivas y no cognitivas entre hombres y mujeres, a cómo influyen estas dotaciones en sus elecciones ocupacionales y, finalmente, a cómo se traducen estas distintas trayectorias en brechas remunerativas importantes.

Hay, por lo menos, dos grandes espacios para la discusión de política pública a partir de este trabajo. Por un lado, se trataría de cómo asegurar la formación temprana (y equitativa) de las habilidades cognitivas y no cognitivas para todos los niños en el Perú. Por otro lado, se debería buscar cómo asegurar una cierta equidad de oportunidades en el acceso a todo tipo de empleos sin distingo de género.

En cuanto al primer tema, la administración educativa actual en el Perú ha puesto como primera prioridad en sus políticas el objetivo de equidad e inclusión social. En su lenguaje concreto, se trata de reducir las brechas de acceso a las oportunidades educativas (Minedu, 2012) que ocurren entre zonas urbanas y rurales, entre diferentes etnias y entre tipos de instituciones. Pero el énfasis aún existe sólo en reducir las grandes diferencias en habilidades cognitivas que muestran los estudiantes. 
El indicador que más se utiliza son los resultados de las pruebas de comprensión de lectura y de habilidad lógico-matemática en niños de segundo grado. Por ejemplo, en la región de Loreto - selva peruana- sólo el 5 por ciento de los niños aprueban esta evaluación censal, mientras que en la región capital de Lima metropolitana el 45 por ciento de los niños lo hacen. En el Perú todavía no se incluye en el currículum oficial de la educación básica el dominio de las competencias blandas tan enfatizadas en este artículo. Felizmente, nuestro reclamo empieza a ser crecientemente compartido por la comunidad empresarial, la que se queja del enorme déficit de estas habilidades en los actuales ingresantes a la fuerza laboral en el país.

En cuanto al segundo tema - promover una mayor equidad de oportunidades en el acceso a todo tipo de empleos sin distingo de género- , también hay mucho camino por recorrer. Una reciente investigación con datos originales de Lima metropolitana (Galarza y Yamada, 2012) encuentra que los postulantes hombres tienen 31 por ciento más de probabilidad que las postulantes mujeres de ser llamados a entrevistas de trabajo, en una muestra de casi cinco mil individuos que respondieron con currículos comparables a avisos de vacantes publicadas en el periódico de mayor circulación local.

A pesar de firmar todos los acuerdos internacionales en materia de políticas antidiscriminación, el Perú ha hecho poco para cumplir con sus compromisos asumidos. Al respecto, podría empezarse con acciones concretas de parte del sector público, tales como la evaluación de currículos anónimos (sin nombres, ni apellidos, ni fotos, ni sexo) en sus actividades de reclutamiento de personal. El Estado también podría promover que las empresas privadas con buenas prácticas laborales y programas de responsabilidad social realicen una primera evaluación de postulantes a través de currículos anónimos y que contraten progresivamente más minorías. Asimismo, el Estado, siendo el comprador individual más grande de la economía, podría preferir adquirir bienes y servicios de parte de empresas que activamente implementen programas de igualdad de oportunidades laborales dentro de sus políticas de recursos humanos. 


\section{REFERENCIAS BIBLIOGRÁFICAS}

Bassi, Marisa \& Sebastian Galiani. 2009. "Labor Market Insertion of Young Adults in Chile". Inter-American Development Bank. Manuscrito no publicado.

Bowles, Samuel \& Herbert Gintis. 1976. Schooling in Capitalist America: Educational Reform and the Contradictions of Economic Life. New York: Basic Books.

Castro, Juan F. \& Gustavo Yamada. 2012. "Convexification and Deconvexification of the Peruavian Wage Profile: A Tale of Declining Education Quality". Working Papers 12-02, Departamento de Economía, Universidad del Pacífico.

Cobb-Clark, Deborah A. \& Michelle Tan. 2009. "Noncognitive Skills, Occupational Attainment, and Relative Wages”. IZA Discussion Papers 4289, Institute for the Study of Labor (IZA).

Cunha, Flavio, James J. Heckman, Lance Lochner \& Dimitriy V. Masterov. 2006. "Interpreting the Evidence on Life Cycle Skill Formation". En Handbook of the Economics of Education, vol 1, editado por Eric. A. Hanushek y Finis Welch. Amsterdam: North Holland.

. 2007. "The Technology of Skill Formation". American Economic Review 97 (2): 31-47.

Cunha, Flavio, James Heckman \& Susanne Schennach. "Estimating the Technology of Cognitive and Noncognitive Skill Formation". Econometrica 78 (3): 883-931.

Díaz, Juan J., Omar Arias \& David Vera Tudela. 2012. "Does Perseverance Pay as Much as Being Smart? The Returns to Cognitive and Non-Cognitive Skills in Urban Peru". Mimeo.

Duckworth Angela, Christopher Peterson, Michael D. Matthews \& Dennis R. Kelly. 2007. "Grit: Perseverance and Passion for Long-Term Goals". Journal of Personality and Social Psychology 92 (6): 1087-1101.

Edwards, Richard C. 1976. "Personal Traits and Success in Schooling and Work". Educational and Psychological Measurement 37: 125-138.

Felices, Guillermo. 1996. "El nuevo papel de la mujer en los mercados de trabajo". En Caminos entrelazados: la realidad del empleo urbano en el Perú, editado por Gustavo Yamada. Lima: Universidad del Pacífico.

Fortin, Nicole M. 2008. "The Gender Wage Gap Among Young Adults in The United States: The Importance of Money Versus People". Journal of Human Resources 43 (4): 884-918.

Galarza, Francisco \& Gustavo Yamada. 2012. "Labor Market Discrimination in Lima, Perú: Evidence from a Field Experiment”. Working Papers 12-03, Departamento de Economía, Universidad del Pacífico.

Goldberg Lewis R. 1990. “An Alternative 'Description of Personality': The BigFive Factor Structure”. Journal of Personality and Social Psychology 59 (6): 1216-1229. doi: 10.1037/0022-3514.59.6.1216.

Grove, Wayne A., Andrew Hussey \& Michael Jetter. 2011. “The Gender Pay Gap Beyond Human Capital: Heterogeneity in Noncognitive Skills and in Labor Market Tastes". Journal of Human Resources 46 (4): 827-874. 
Hansen, Karsten T., James J. Heckman \& J. K. Kathleen J. Mullen. 2004. "The Effect of Schooling and Ability on Achievement Test Scores". Journal of Econometrics 121 (1-2): 39-98.

Heckman, James J., Jora Stixrud \& Sergio Urzúa. 2006. “The Effects of Cognitive and Noncognitive Abilities on Labor Market Outcomes and Social Behavior". Journal of Labor Economics 24 (2): 411-482.

Helmers, Christian \& Manasa Patnam. 2011. "The Formation and Evolution of Childhood Skill Acquisition: Evidence from India”. Journal of Development Economics 95 (2): 252-266.

Herrnstein, Richard J. \& Charles Murray. 1994. The Bell Curve: Intelligence and Class Structure in American Life. New York: Free Press.

Klein, Roger, Richard Spady \& Andrew Weiss. 1991. "Factors Affecting the Output and Quit Propensities of Production Workers". Review of Economic Studies 58 (2): 929-954.

Murnane, Richard J., Frank Levy \& John B. Willett. 1995. "The Growing Importance of Cognitive Skills in Wage Determination". Review of Economics and Statistics 77 (2): 251-266.

Neal, Derek A. \& William R. Johnson. 1996. "The Role of Premarket Factors in Black-White Wage Differences". Journal of Political Economy 104 (5): 869895.

Ñopo, Hugo. 2009. "The Gender Wage Gap in Peru 1986-2000: Evidence from a Matching Comparisons Approach”. Research Department Publications 4618, Inter-American Development Bank.

Ritter, Joseph A. \& Lowell J. Taylor. 2011. "Racial Disparity in Unemployment". The Review of Economics and Statistics 93 (1): 30-42.

Urzúa, Sergio, David Bravo \& Claudia Sanhueza. 2009. “Ability, Schooling Choices and Gender Labor Market Discrimination: Evidence for Chile". En Discrimination in Latin America: An Economic Perspective, editado por Alberto Chong, Andrea Moro y Hugo Nopo. Washington, D.C.: World Bank Publications. EP 